\title{
Astrometric and Timing Effects of Gravitational Waves from Localized Sources
}

\author{
Sergei M. Kopeikin* and Gerhard Schäfer \\ Theoretisch-Physikalisches Institut der Friedrich-Schiller-Universität Jena, Max-Wien-Platz 1, 07743 Jena, Germany \\ Carl R. Gwinn \\ Department of Physics, Broida Hall, University of California at Santa Barbara, Santa Barbara, CA 93106
}

T. Marshall Eubanks

US Naval Observatory, 3450 Massachusetts Avenue, Washington, DC 20392

\section{Contents}

\begin{tabular}{ll}
\hline I Introduction & 3 \\
\hline
\end{tabular}

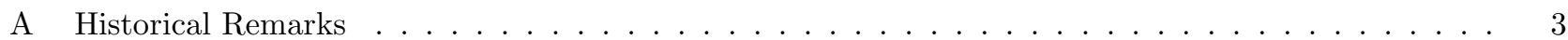

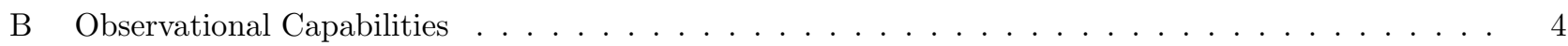

\begin{tabular}{llr}
\hline II $\quad$ Equations of propagation of electromagnetic waves & 4
\end{tabular}

\begin{tabular}{|lr}
\hline III $\quad$ Metric Tensor and Coordinate Systems & 5
\end{tabular}

\begin{tabular}{ll|} 
IV Method of Integration of the Equations of Motion & 8
\end{tabular}

A $\quad$ Useful Relationships . . . . . . . . . . . . . . . . . . . . . . . . . . . . . 8

B Calculation of Integrals from the Static Part of the Gravitational Field . . . . . . . . . . . . . . . 10

C Calculation of Integrals from Time Dependent Part of Gravitational Field . . . . . . . . . . . . . 11

1 First Method of Integration $\ldots \ldots \ldots \ldots \ldots \ldots \ldots$

2 Second Method of Integration $\ldots \ldots \ldots \ldots \ldots$

\begin{tabular}{llr}
\hline V Perturbations of Photon's Trajectory & 15
\end{tabular}

\begin{tabular}{lll}
\hline VI $\quad$ Basic Observable Relativistic Effects & 17
\end{tabular}

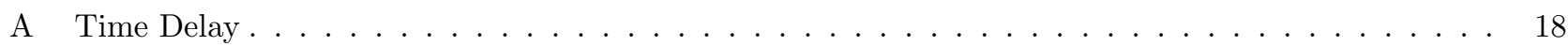

B Deflection of Light . . . . . . . . . . . . . . . . . . . . . . . . . . . . . . 19

\begin{tabular}{ll}
\hline VII Discussion & 22 \\
\hline
\end{tabular}

A Case 1. Small Impact Parameter $\left(\tau_{0}<0\right) \ldots \ldots \ldots \ldots \ldots \ldots \ldots \ldots \ldots \ldots \ldots$

1 Asymptotic expansions of independent variables $\ldots \ldots \ldots \ldots \ldots \ldots \ldots$

2 Asymptotic expressions for time delay and the angle of light deflection . . . . . . . . . . 25

B Case 2. Small Impact Parameter $\left(\tau_{0}>0\right) \ldots \ldots \ldots \ldots \ldots \ldots \ldots \ldots \ldots$

1 Asymptotic expansions of independent variables $\ldots \ldots \ldots \ldots \ldots \ldots \ldots \ldots$

2 Asymptotic expressions for time delay and the angle of light deflection . . . . . . . . . . . . 29

C Case 3. Large Impact Parameter . . . . . . . . . . . . . . . . . . . . . . . . . . 30

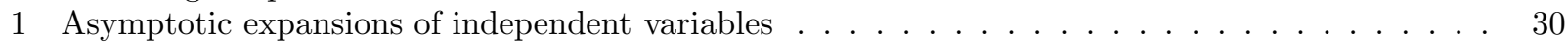

2 Asymptotic expressions for time delay and the angle of light deflection $\ldots \ldots \ldots$. . . . . . 31

\begin{tabular}{ll}
\hline VIII Conclusions & 33
\end{tabular}

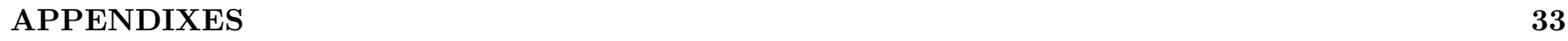

\begin{tabular}{|ll|}
\hline A Comparison to the paper by Damour \& Esposito-Farèse & 33
\end{tabular}

B Harmonic and ADM gauge conditions in the first post-Minkowskian approximation 35

*On leave from: ASC FIAN, Leninskii Prospect, 53, Moscow, 117924, Russia 
The extremely high precision of current radio interferometric observations demands a better theoretical treatment of secondary effects in the propagation of electromagnetic signals in variable gravitational fields. Such fields include those of oscillating and precessing stars, stationary or coalescing binary systems, and colliding galaxies. Especially important is the problem of propagation of light rays in the field of gravitational waves emitted by a localized source of gravitational radiation. A consistent approach for a complete and exhaustive solution of this problem is developed in the present paper in the first post-Minkowskian and quadrupole approximation of General Relativity. This approximation is linear with respect to the universal gravitational constant $G$ and accounts for the static monopole, spin, and time-dependent quadrupole moments of an isolated system. We demonstrate for the first time that the equations of light propagation in the retarded gravitational field of an arbitrary localized source emitting quadrupolar gravitational waves can be integrated exactly in closed form. The influence of the gravitational field under consideration on the light propagation is examined not only in the wave zone but also in cases when light passes through the intermediate and near zones of the source. We reproduce the known results of integration of equations of light rays, both in a stationary gravitational field and in the field of plane gravitational waves, establishing the relationship between our new formalism and the simplified approaches of other authors. Explicit analytic expressions for light deflection and integrated time delay (Shapiro effect) are obtained accounting for all possible retardation effects and arbitrary relative locations of the source of gravitational waves, the source of light rays, and the observer. Coordinate dependent terms in the expressions for observable quantities are singled out and used for physically meaningful interpretation of observable quantities. It is shown that the ADM and harmonic gauge conditions can both be satisfied simultaneously outside the source of gravitational waves. Such ADM-harmonic coordinates are extensively used in the present paper. Their use drastically simplifies the integration of light propagation equations and the equations for the motion of light source and observer in the gravitational field of the source of gravitational waves, leading to the unique interpretation of observable effects. The two limiting cases of small and large values of impact parameter $d$ are elaborated in more detail. It is proved that leading order terms for the effect of light deflection in the case of small impact parameter depend neither on the radiative part $(\sim 1 / d)$ of the gravitational field nor on the intermediate $\left(\sim 1 / d^{2}\right)$ zone terms, confirming a previous result in the literature. The main effect rather comes from the near zone $\left(\sim 1 / d^{3}\right)$ terms. This property of strong suppression of the influence of gravitational waves on the propagation of light rays makes much more difficult any direct detection of gravitational waves by VLBI or pulsar timing techniques, in contrast to previous claims by other authors. We also present a thorough-going analytical treatment of time delay and bending of light in the case of large impact parameter. This exploration essentially extends previous results regarding propagation of light rays in the field of a plane monochromatic gravitational wave. Explicit expressions for Shapiro effect and deflection angle are obtained in terms of the transversetraceless (TT) part of the space-space components of the metric tensor. We also discuss the relevance of the developed formalism for interpretation of radio interferometric and timing observations, as well as for data processing algorithms for future gravitational wave detectors. 


\section{INTRODUCTION}

\section{A. Historical Remarks}

Binary systems are well known sources of periodic gravitational waves. Indirect proof of the existence of gravitational waves emitted by binary pulsars was given by Taylor [1]. However, the direct observation of gravitational waves still remains the unsolved problem of experimental gravitational physics. The expected spectrum of gravitational waves extends from $\sim 10^{4} \mathrm{~Hz}$ to $10^{-18} \mathrm{~Hz}$ [2], 3]. Within that range, the spectrum of periodic waves from known binary systems extends from about $10^{-3} \mathrm{~Hz}$, the frequency of gravitational radiation from a contact white-dwarf binary [ 4 , through the $10^{-4}$ to $10^{-6} \mathrm{~Hz}$ range of radiation from main-sequence binaries [5], to the $10^{-7}$ to $10^{-9} \mathrm{~Hz}$ frequencies emitted by binary supermassive black holes postulated to lie in galactic nuclei [6]. The dimensionless strain of these waves at the Earth, $h$, may be as great as $10^{-21}$ at the highest frequencies, and as great as $3 \times 10^{-15}$ at the lowest frequencies in this range.

Sazhin [7] first suggested detection of gravitational waves from a binary system using timing observations of a pulsar, the line of sight to which passes near the binary. It was shown that the integrated time delay for propagation of the electromagnetic pulse near the binary is proportional to $1 / d^{2}$ where $d$ is the impact parameter of the unperturbed trajectory of the signal. More recently, Sazhin \& Saphonova [8] made estimates of the probability of observations of this effect, for pulsars in globular clusters, and showed that the probability can be high, reaching 0.97 for one cluster. We note however that mathematical technique worked out in these papers allows rigorous treatment only of effects of the near-zone, quasi-static quadrupolar part of the gravitational field and is not enough to make any conclusion about actual observability of gravitational waves emitted by a binary system.

Wahlquist [9] made another approach to the detection of periodic gravitational waves, based on Doppler tracking of spacecraft traveling in deep space. His approach is restricted by the plane gravitational wave approximation developed earlier by Estabrook \& Wahlquist [10]. Tinto ( [11], and references therein) made the most recent theoretical contribution in this area. The Doppler tracking technique has been used in space missions, by seeking the characteristic triple signature, the presence of which would reveal the influence of a gravitational wave crossing the line of sight from spacecraft to observer [12].

Quite recently, Braginsky et al. [13] (see also [14) have raised the question of using astrometry as a detector of stochastic gravitational waves. This idea has also been investigated by Kaiser \& Jaffe [15] and, in particular, by Pyne et al. [16] and Gwinn et al. [17] who showed that the overall effect is proportional to the strain of metric perturbation caused by the plane gravitational wave and set observational limits on the energy density of ultra long gravitational waves present in early universe. Montanari [18] studied polarization perturbations of free electromagnetic radiation in the field of a plane gravitational wave and found that the effects are exceedingly small.

Fakir ( [19], and references therein) has suggested the possibility of using astrometry to detect periodic variations in apparent angular separations of appropriate light sources, caused by gravitational waves emitted from isolated sources of gravitational radiation. He was not able to develop a self-consistent approach to tackle the problem with necessary completeness and rigor. For this reason his estimate of the effect is erroneous. Another attempt to work out a more consistent approach to the calculation of the deflection angle in the field of arbitrary source of gravitational waves has been undertaken by Durrer [20]. However, the calculations have been done only for the plane wave approximation and the result obtained was extrapolated for the case of the localized source of gravitational waves without justification. For this reason the deflection angle was overestimated. The same misinterpretation of the effect can be found in the paper by Labeyrie [21] who studied a photometric modulation of background sources by gravitational waves emitted by fast binary stars. Because of this, the expected detection of the gravitational waves from the observations of the radio source GPS QSO 2022+171 suggested by Pogrebenko et al. [22] was not based on firm theoretical ground.

Damour \& Esposito-Farèse [23] have studied the deflection of light and integrated time delay caused by the timedependent gravitational field generated by a localized material source lying close to the line of sight. They explicitly took into account the full, retarded gravitational field in the near, intermediate, and wave zones. Contrary to the claims of Fakir [19] and Durrer [20] and in agreement with Sazhin's [7] calculations, they found that the deflections due to both the wave-zone gravitational wave and the intermediate-zone retarded fields vanish exactly. The leading total time-dependent deflection is given only by the quasi-static, near-zone quadrupolar piece of the gravitational field.

In the present paper we work out an even more systematic approach to the problem. While Damour \& EspositoFarèse 23] considered both the light source and the observer to be located at infinity, and performed their calculations in terms of the spacetime Fourier transform, we do not need these assumptions. Our approach is much more general and applicable for any location of the source of light and observer in space with respect to the source of gravitational radiation. The integration technique which we use for finding the solution of equations of propagation of light rays was partially employed in 24 and does not require any implementation of the spacetime Fourier transform. 
Section 2 of the present paper discusses equations of propagation of electromagnetic waves in the geometric optics approximation. The metric tensor and coordinate systems involved in our calculations are described in section 3 along with gauge conditions imposed on the metric tensor. The method of integration of the equations of motion with emphasis on specific details of calculations of particular integrals is given in section 4. Exact solution of the equations of light propagation and the form of relativistic perturbations of the light trajectory are obtained in section 5 . Section 6 is devoted to derivation of basic observable relativistic effects - the integrated time delay and the deflection angle. We find the more precise meaning of quite general formulae obtained in the previous section by discussing in section 7 several limiting cases in the relative configuration of the source of light, the observer, and the source of gravitational waves. Section 8 contains concluding remarks. Appendix A compares results of our calculations with those by Damour \& Esposito-Farèse [23] and proves their gauge invariance. Appendix B gives more details on the derivation of the ADM-harmonic coordinate system used in the present paper for interpretation of observed relativistic effects.

\section{B. Observational Capabilities}

Calculations of the effects of gravitational waves are of most interest if they indicate that those can be detected with present techniques, or foreseeable improvements. Astrometric precision and accuracy have evolved rapidly in the last decades, and can be expected to continue to improve. In principle, the accuracy attainable with a given instrument is approximately the angular resolution of the instrument, divided by the signal-to-noise ratio. In practice, the time span of the observations and the angular separation of the source from reference sources critically affect the attainable accuracy.

Very-long baseline interferometry, or VLBI, attains the highest angular resolution available on an operational basis. It achieves angular resolution set by the diffraction limit, of $\Delta \theta \approx \lambda / B$, where $B$ is the separation of the interferometer elements (the baseline), and $\lambda$ is the observing wavelength. Practical baselines may be about as long as an Earth radius, $B \sim 6400 \mathrm{~km}$; a typical observing wavelength is $\lambda=3 \mathrm{~cm}$, yielding angular resolution of 1 milliarcsecond.

Observations of a moderately strong $(\sim 1 \mathrm{Jy})$ extragalactic source, such as a quasar, can reach signal-to-noise ratio of several hundred in 5 or 10 minutes, offering potential angular accuracy of microarcseconds. In principle, a day of integration with the US Very Long Baseline Array (VLBA) could yield angular accuracy of about 0.1 microarcseconds.

Observations using the largest radiotelescopes can increase the signal-to-noise ratio by a factor of $\sim 10 \times$. In practice, a host of geodetic and propagation effects limit the reproducibility of VLBI astrometry. These factors must either be measured during the observations, or calculated from models. At present, atmospheric stability and changes in source structure limit reproducibility of measured angles between sources to about 1 milliarcsecond, over periods of months. Observations of pairs of radio sources, with separations of $\sim 0.5^{\circ}$, can yield angular accuracy of about 50 microarcseconds, reproducible over periods of years, when effects of source structure are included 25.

Astrophysical $\mathrm{H}_{2} \mathrm{O}$ masers have extremely high flux densities, of up to $10^{6} \mathrm{Jy}$ at $\lambda=1.3 \mathrm{~cm}$. In principle, a day of observation of masers with the VLBA could yield angular accuracy of a few picoarcseconds. Observations of masers have attained reproducibility of better than 10 microarcseconds over several months, between individual maser spots in a Galactic maser cluster, with separations of a few arcsec 26]. Astrometric observations of extragalactic masers have attained accuracies of better than 1 microarcsecond, for maser spots separated by less than 1 arcsec [27. Atmospheric variations probably dominate the error budget.

Shorter wavelengths offer potentially higher diffraction-limited angular resolution, but practical obstacles are severe. Atmospheric effects present greater phase changes, on shorter time scales; and photon shot noise becomes a limiting factor for fainter sources and at shorter wavelengths. Optical interferometers in space will probably equal and exceed the accuracy of VLBI. For example, the Space Interferometry Mission (SIM), and the proposed European mission GAIA seek to attain angular accuracy of about 1 microarcsecond in several hours of integration [28] - 30].

Astrometric observations to seek effects of gravitational waves could attain higher accuracy, at least on shorter timescales. The periods of the waves, and of the expected deflection, could be short enough to avoid some atmospheric and other propagation effects. For known binary systems, the wave period, and perhaps the phase, are known accurately, permitting search for deflections at this period. Such a "synchronous" search would eliminate many noise sources, allow detection of short-period motions with the sensitivity resulting from long integrations, and perhaps allow astrometric accuracy to approach the signal-to-noise ratio limit.

\section{EQUATIONS OF PROPAGATION OF ELECTROMAGNETIC WAVES}

We assume the approximation of geometric optics, as the wavelength of electromagnetic waves used for astrometric observations is usually much smaller than wavelength of gravitational waves emitted by isolated astronomical systems 
like binary stars or supernova explosions [2]. This allows us to use the relativistic equation of geodesic motion of a massless particle (such as a photon) for description of the process of propagation of electromagnetic signal from the source of light to the observer at the Earth. We also assume that space-time is asymptotically flat. This assumption does not hold for cosmological distances. However, if we neglect all terms depending on the rate of cosmological expansion and make a rescaling of time and space coordinates with the cosmological scale factor $a(t)$, our formalism will be still valid for application in cosmology.

We denote spatial coordinates by $x^{i}=\mathbf{x}=\left(x^{1}, x^{2}, x^{3}\right)$ and time coordinate $x^{0}=c t$, where $c$ is the speed of light and $t$ is coordinate time. Let the motion of a photon be defined by fixing the mixed initial-boundary conditions introduced and extensively used by Brumberg [31]

$$
\mathbf{x}\left(t_{0}\right)=\mathbf{x}_{0}, \quad \frac{d \mathbf{x}(-\infty)}{d t}=\mathbf{k},
$$

where $\mathbf{k}^{2}=1$ and the spatial components of vectors are denoted by bold letters. These conditions define the coordinates $\mathbf{x}_{0}$ of the photon at the moment of emission $t_{0}$ and its velocity at the infinite past and the infinite distance from the origin of the spatial coordinates (that is, at past null infinity). In what follows we put $c=1$ for convenience.

Equation of propagation of photons in a weak gravitational field is given in the first post-Minkowskian approximation by the formula 31,32 :

$$
\begin{aligned}
\ddot{x}^{i}(t)= & \frac{1}{2} g_{00, i}-g_{0 i, t}-\frac{1}{2} g_{00, t} \dot{x}^{i}-g_{i k, t} \dot{x}^{k}-\left(g_{0 i, k}-g_{0 k, i}\right) \dot{x}^{k}- \\
& g_{00, k} \dot{x}^{k} \dot{x}^{i}-\left(g_{i k, j}-\frac{1}{2} g_{k j, i}\right) \dot{x}^{k} \dot{x}^{j}+\left(\frac{1}{2} g_{k j, t}-g_{0 k, j}\right) \dot{x}^{k} \dot{x}^{j} \dot{x}^{i},
\end{aligned}
$$

where the $g_{00}, g_{0 i}, g_{i j}$ are components of metric tensor, fully determined by the given distribution and motion of mass inside the source of gravitational field, dots over vectors denote the total derivative with respect to time, and commas indicate partial derivatives with respect to spatial or time coordinates; that is, for any function $f_{, i}=\partial f / \partial x^{i}$, $f_{, t}=\partial f / \partial t$. Hereafter repeated latin indices mean summation from 1 to 3 . The given equation is valid in arbitrary coordinates (gauges) and represents the ordinary second order differential equation for light propagation.

The right-hand side of equation (2) includes terms which depend on the coordinate velocity $\dot{x}^{i}$ of the photon, in the weak-field approximation approximately equal to the speed of light $c$. We restrict ourselves to finding a solution of equation (2) only in the first linear approximation with respect to the universal gravitational constant $G$. For this reason, when solving equation (2), only one iteration is enough and it is admissible to make the replacement $\dot{x}^{i}=k^{i}$ in the right-hand side of the equation. The result of this approach is:

$$
\begin{aligned}
\ddot{x}^{i}(t)= & \frac{1}{2} g_{00, i}-g_{0 i, t}-\frac{1}{2} g_{00, t} k^{i}-g_{i j, t} k^{j}-\left(g_{0 i, j}-g_{0 j, i}\right) k^{j}- \\
& \mathrm{g}_{00, j} k^{j} k^{i}-\left(g_{i p, j}-\frac{1}{2} g_{p j, i}\right) k^{p} k^{j}+\left(\frac{1}{2} g_{p j, t}-g_{0 p, j}\right) k^{p} k^{j} k^{i} .
\end{aligned}
$$

This equation must be solved to obtain a perturbed trajectory of the photon propagating through the gravitational field of an isolated astronomical system emitting gravitational waves. To accomplish this task one needs a mathematical expression for the metric tensor.

\section{METRIC TENSOR AND COORDINATE SYSTEMS}

Let us chose the origin of the asymptotically flat coordinate frame at the center of mass-energy of the isolated astronomical system and impose the de-Donder (harmonic) gauge conditions on components of the "canonical" metric tensor. We assume that gravitational field is weak and the metric of spacetime $g_{\alpha \beta}$ is written as a sum of the Minkowski metric $\eta_{\alpha \beta}=\operatorname{diag}(-1,1,1,1)$ plus a small perturbation $h_{\alpha \beta}$ :

$$
g_{\alpha \beta}=\eta_{\alpha \beta}+h_{\alpha \beta},
$$

where the Greek indices run from 0 to 3. The most general expression for the linearized metric tensor, generated by a system emitting gravitational waves, in terms of its symmetric and trace-free (STF) mass and spin multipole moments is given by Thorne [33] (see also [34,35]). It can be written as 


$$
h_{\alpha \beta}=h_{\alpha \beta}^{c a n}+\nabla_{\beta} w_{\alpha}+\nabla_{\alpha} w_{\beta},
$$

where $\nabla_{\alpha}=\partial / \partial x^{\alpha}$. The "canonical" form of the metric tensor perturbations in harmonic gauge reads as follows [36]

$$
\begin{aligned}
h_{00}^{\text {can. }}= & \frac{2 \mathcal{M}}{r}+2 \sum_{l=2}^{\infty} \frac{(-1)^{l}}{l !}\left[\frac{\mathcal{I}_{A_{l}}(t-r)}{r}\right]_{, A_{l}}, \\
h_{0 i}^{c a n .}= & -\frac{2 \epsilon_{i p q} \mathcal{S}_{p} N_{q}}{r^{2}}- \\
& 4 \sum_{l=2}^{\infty} \frac{(-1)^{l} l}{(l+1) !}\left[\frac{\epsilon_{i p q} \mathcal{S}_{p A_{l-1}}(t-r)}{r}\right]_{, q A_{l-1}}+4 \sum_{l=2}^{\infty} \frac{(-1)^{l}}{l !}\left[\frac{\dot{\mathcal{I}}_{i A_{l-1}}(t-r)}{r}\right]_{, A_{l-1}}, \\
h_{i j}^{c a n .}= & \delta_{i j}\left\{\frac{2 \mathcal{M}}{r}+2 \sum_{l=2}^{\infty} \frac{(-1)^{l}}{l !}\left[\frac{\mathcal{I}_{A_{l}}(t-r)}{r}\right]_{, A_{l}}\right\}+ \\
& 4 \sum_{l=2}^{\infty} \frac{(-1)^{l}}{l !}\left[\frac{\ddot{\mathcal{I}}_{i j A_{l-2}}(t-r)}{r}\right]_{, A_{l-2}}-8 \sum_{l=2}^{\infty} \frac{l(-1)^{l} l}{(l+1) !}\left[\frac{\epsilon_{p q(i} \dot{\mathcal{S}}_{j) p A_{l-2}}(t-r)}{r}\right]_{, q A_{l-2}} .
\end{aligned}
$$

where $N^{i}=x^{i} / r$, and the round brackets around indices in equation (8) means symmetrization; that is, for any two indices $T_{(i j)}=\frac{1}{2}\left(T_{i j}+T_{j i}\right)$. In the pure harmonic gauge the functions $w^{0}, w^{i}$ are solutions of the homogeneous d'Alembert's equation and are given by the expressions

$$
\begin{aligned}
& w^{0}=\sum_{l=0}^{\infty}\left[\frac{\mathcal{W}_{A_{l}}(t-r)}{r}\right]_{, A_{l}}, \\
& w^{i}=\sum_{l=0}^{\infty}\left[\frac{\mathcal{X}_{A_{l}}(t-r)}{r}\right]_{, i A_{l}}+\sum_{l=1}^{\infty}\left\{\left[\frac{\mathcal{Y}_{i A_{l-1}}(t-r)}{r}\right]_{, A_{l-1}}+\left[\epsilon_{i p q} \frac{\mathcal{Z}_{q A_{l-1}}(t-r)}{r}\right]_{, p A_{l-1}}\right\},
\end{aligned}
$$

where $\mathcal{W}_{A_{l}}, \mathcal{X}_{A_{l}}, \mathcal{Y}_{i A_{l-1}}$, and $\mathcal{Z}_{q A_{l-1}}$ are arbitrary functions of time. Their specific choice will be made later on in the discussion regarding the interpretation of observable effects. In equations (6)-(10), we adopt the notation: $A_{l}=a_{1} a_{2} \ldots a_{l}$ is a polyindex, $\mathcal{M}$ is the total (Tolman or ADM) mass of the system, $\mathcal{I}_{A_{l}}$ and $\mathcal{S}_{A_{l}}$ are the STF mass and spin gravitational multipoles, and $\mathcal{W}_{A_{l}}, \mathcal{X}_{A_{l}}, \mathcal{Y}_{A_{l}}, \mathcal{Z}_{A_{l}}$ are multipoles which reflect the freedom of coordinate transformations. These multipoles can be eliminated from the metric using the transformation

$$
x^{\prime \alpha}=x^{\alpha}-w^{\alpha},
$$

relating an original harmonic coordinate system $x^{\alpha}$ to another harmonic one $x^{\prime \alpha}$, in which only the "canonical" part of the metric is present.

However, we would like to emphasize that, in general, equation (5) holds in an arbitrary gauge. Particular examples of functions $w^{0}$ and $w^{i}$ in harmonic gauge are given in equations (9)-(10). Other expressions for $w^{0}$ and $w^{i}$ in the ADM (Arnowitt-Deser-Misner) gauge [37] are given in Appendix B wherein we also prove that it is possible to choose functions $w^{0}$ and $w^{i}$ in such a way that $\mathrm{ADM}$ and harmonic gauge conditions will be satisfied simultaneously. This means that the classes of harmonic and ADM coordinates overlap. The discussion of different gauges is helpful for giving a unique interpretation of observable effects by properly fixing the coordinate degrees of freedom in corresponding quantities [38].

The STF cartesian tensor has a special algebraic structure which eliminates all reducible parts of the tensor and leaves only the irreducible part having the highest rank [33,40]. In other words, contraction over of any two indices of STF tensor gives identically zero. It is worth noting the absence of the dipole mass multipole $\mathcal{I}_{i}$ in equations (6)-(8) which is identically zero, due to the choice of the origin of coordinate system at the center of mass of the gravitating system. We also stress that the multipoles in the linearized metric (6)-(10) depend on the "retarded time" $t-r$. At first sight this dependence seems to make subsequent calculations more difficult. However, just the opposite happens and the dependence of the multipoles on the retarded time makes the calculations simpler. 
In what follows we consider the concrete case of a localized deflector emitting gravitational waves. In this section we restrict ourselves to considering the influence of gravitational field of the deflector on the propagation of electromagnetic signals made by its total constant mass $M$, spin $\mathcal{S}$, and time-dependent quadrupole moment $\mathcal{I}_{i j}(t-r)$ only. This simplifies the expressions (6)-(8) for the metric tensor, which are reduced to the expressions

$$
\begin{aligned}
& h_{00}^{c a n .}=\frac{2 \mathcal{M}}{r}+\nabla_{p} \nabla_{q}\left[\frac{\mathcal{I}_{p q}(t-r)}{r}\right], \\
& h_{0 i}^{c a n .}=-\frac{2 \epsilon_{i p q} \mathcal{S}_{p} N_{q}}{r^{2}}+2 \nabla_{j}\left[\frac{\dot{\mathcal{I}}_{i j}(t-r)}{r}\right], \\
& h_{i j}^{c a n .}=\delta_{i j} h_{00}^{c a n .}+q_{i j}^{c a n .},
\end{aligned}
$$

where

$$
q_{i j}^{c a n .}=\frac{2}{r} \ddot{\mathcal{I}}_{i j}(t-r) .
$$

Herein terms depending on $\mathcal{M}$ and $\mathcal{S}_{i}$ are static and produce well-known effects in the propagation of light rays. Retarded terms that are inversely proportional to the distance $r$ from the gravitating system describe the pure gravitational-wave part of the metric.

Let us stress that in the harmonic coordinate system the gravitational-wave part of the metric tensor is present in all of its components and is expressed through the second time derivative of the quadrupole moment [33]. If we choose

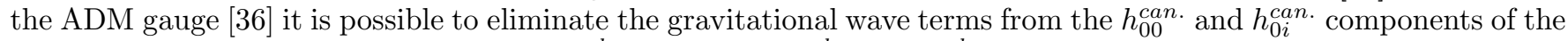
metric tensor and to bring all of them to $h_{i j}^{a d m}$ [41]. Then $h_{00}^{a d m}$ and $h_{0 i}^{a d m}$ depend only on the "instantaneous" time $t$ and not on the retarded time $t-r$ (see Appendix B). In combining the ADM gauge with the harmonic gauge an even simpler representation is possible where $h_{00}$ and $h_{0 i}$ do not depend on time at all. However, the transformation from the canonical form of metric (12)-(15) to the ADM-harmonic form includes integration of the quadrupole moment with respect to time. Appendix B gives a more detailed study of this procedure.

One might ask whether the ADM or harmonic coordinate system is more preferable, for the adequate physical treatment of the relativistic time delay and deflection of light rays in the field of gravitational waves emitted by a localized source. Our point of view is that the coordinate system should be chosen in such a way to be simultaneously both ADM and harmonic. The reason for this is that an observer who is at rest with respect to the ADM coordinate system does not feel the gravitational force caused by gravitational waves. This means that if the instantaneous gravitational field of the localized source may be neglected, the observer fixed with respect to the ADM system can be considered to be in free fall. Hence, no artificial force need be applied to the observer in order to keep him at rest at the fixed coordinate point. The motion of such an observer is described by the extremely simple equation $x^{i}=$ const and there is no need to account for kinematic effects associated with the observer's motion. All these advantages are lost in the "canonical" harmonic gauge. An observer fixed with respect to that coordinate system must be kept at a fixed coordinate point by some external force to prevent his motion under the influence of gravitational waves. The existence of such a force is unnatural from physical and astronomical points of view. On the other hand, the "canonical" harmonic gauge has the advantage of a much simpler integration of the equations of light propagation than the "canonical" ADM gauge. One can see that the "canonical" ADM metric coefficients (B1)-(B2) contain functions which depend on time $t$ only. As will be clear from the procedure of integration of equations of light propagation described in the next section such "instantaneous" functions of time do not permit explicit integration of each specific term (only after summing all terms is the explicit integration possible). Fortunately, the classes of ADM and harmonic coordinate systems overlap and, for this reason, we can substantially benefit by choosing a coordinate system that is simultaneously both ADM and harmonic. This allows us to proceed in the following way. First we integrate equations of light propagation in the harmonic gauge and then apply coordinate transformations (B13)-(B14) which transform the pure harmonic coordinate system to the ADM one without violation of the harmonic gauge conditions. This simplifies the treatment of observable effects drastically. 


\section{METHOD OF INTEGRATION OF THE EQUATIONS OF MOTION}

\section{A. Useful Relationships}

We introduce astronomical coordinates $\mathbf{x} \equiv x^{i}=\left(x^{1}, x^{2}, x^{3}\right)$ corresponding to the plane of the sky of the observer and based on a triad of the unit vectors $\left(\mathbf{I}_{0}, \mathbf{J}_{0}, \mathbf{K}_{0}\right)$. The vector $\mathbf{K}_{0}$ points from the observer toward the deflector, and the vectors $\mathbf{I}_{0}$ and $\mathbf{J}_{0}$ lie in the plane of the sky, being orthogonal to vector $\mathbf{K}_{0}$. The vector $\mathbf{I}_{0}$ is directed to the east, and $\mathbf{J}_{0}$ points towards the north celestial pole. The origin of the coordinate system is chosen to lie at the barycenter of the deflector which emits gravitational waves (see Figure 1).

Another reference frame based on a triad of the unit vectors $(\mathbf{I}, \mathbf{J}, \mathbf{K})$ rotated with respect to vectors $\left(\mathbf{I}_{0}, \mathbf{J}_{0}, \mathbf{K}_{0}\right)$ is useful as well. The vector $\mathbf{K}$ points from the observer toward the source of light, and the vectors $\mathbf{I}$ and $\mathbf{J}$ lie in the plane of the sky, being orthogonal to vector $\mathbf{K}$, which is different from the plane of the sky being orthogonal to vector $\mathbf{K}_{0}$. This is because the "plane of the sky" is actually a sphere, and vectors $\mathbf{K}$ and $\mathbf{K}_{0}$ point in different directions. Mutual orientation of one triad with respect to another one is determined by the following equations

$$
\begin{aligned}
\mathbf{I}_{0} & =\mathbf{I} \cos \Omega+\mathbf{J} \sin \Omega, \\
\mathbf{J}_{0} & =-\mathbf{I} \cos \theta \sin \Omega+\mathbf{J} \cos \theta \cos \Omega+\mathbf{K} \sin \theta, \\
\mathbf{K}_{0} & =\mathbf{I} \sin \theta \sin \Omega-\mathbf{J} \sin \theta \cos \Omega+\mathbf{K} \cos \theta,
\end{aligned}
$$

where rotational angles $\Omega$ and $\theta$ are constant.

To integrate the equations of propagation of electromagnetic waves in curved space-time we must resort to an approximation method. In the Newtonian approximation, the unperturbed trajectory of the light ray is a straight line:

$$
x^{i}(t)=x_{N}^{i}(t)=x_{0}^{i}+k^{i}\left(t-t_{0}\right)
$$

where $t_{0}$ is the instant of time of the photon emission from the point with spatial coordinates $x_{0}^{i}$, and $k^{i}=\mathbf{k}$ is a constant unit vector tangent to the unperturbed trajectory and directed from the point of emission to the point of observation of photon (the vector $\mathbf{k} \approx-\mathbf{K}$ ). In the Newtonian approximation, the coordinate speed of the photon $\dot{x}^{i}=k^{i}$ and is considered to be constant. 24

It is convenient to introduce a new independent parameter $\tau$ along the photon's trajectory according to the rule

$$
\tau \equiv \mathbf{k} \cdot \mathbf{x}=t-t_{0}+\mathbf{k} \cdot \mathbf{x}_{0}
$$

where the dot symbol between two vectors denotes the Euclidean dot product of two vectors. The moment $t_{0}$ of the signal's emission corresponds to the numerical value of the parameter $\tau_{0}=\mathbf{k} \cdot \mathbf{x}_{0}$, and the moment $t^{*}$ of the closest approach of the unperturbed trajectory of the photon to the origin of the coordinate system corresponds to the value $\tau=0$ (note that $\tau_{0}<0$ if the source of light is behind the localaized source of gravitational waves). Thus, we find

$$
\tau=t-t^{*}, \quad \tau_{0}=t_{0}-t^{*}
$$

The variable $\tau$ is negative from the point of emission up to the point of the closest approach, and is positive otherwise. The differential identity $d t=d \tau$ is valid and for this reason the integration along ray's path with respect to time $t$ can be replaced by the integration with respect to parameter $\tau$. Using parameter $\tau$, the equation of the unperturbed trajectory of light ray can be represented as

$$
x^{i}(\tau)=x_{N}^{i}(\tau)=k^{i} \tau+\xi^{i}
$$

and the distance, $r$, of the photon from the origin of coordinate system is given by

$$
r=r_{N}(\tau)=\sqrt{\tau^{2}+d^{2}}
$$

where the length of the constant (for a chosen light ray) transverse vector $\boldsymbol{\xi}=\mathbf{k} \times\left(\mathbf{x}_{0} \times \mathbf{k}\right)=\mathbf{k} \times(\mathbf{x} \times \mathbf{k})$ is called the impact parameter of the unperturbed trajectrory of the light ray, $d=|\boldsymbol{\xi}|$, and the symbol " $\times$ " between two vectors denotes the Euclidean cross product. It is worth emphasizing that the vector $\xi^{i}$ is directed from the origin of the coordinate system toward the point of the closest approach of the unperturbed path of light ray to that origin. The relations 


$$
r+\tau=\frac{d^{2}}{r-\tau}, \quad r_{0}+\tau_{0}=\frac{d^{2}}{r_{0}-\tau_{0}},
$$

also hold, and they are useful for presenting the results of integration of the light ray equations in different form. In particular, if we assume the strong inequalities $d \ll r$, and $d \ll r_{0}$ to hold, then

$$
\tau=r-\frac{d^{2}}{2 r}+\ldots, \quad \tau_{0}=-r_{0}+\frac{d^{2}}{2 r_{0}}+\ldots
$$

which clearly shows that at the moment of light reception $\tau$ is positive and at that of light emission $\tau_{0}$ is negative.

Let us consider a set of curves $x^{i}(\tau)=k^{i} \tau+\xi^{i}$ with different values of vectors $k^{i}$ and $\xi^{i}$. The vector field $k^{i}$, defined along the curve $x^{i}(\tau)$, describes the direction of a bundle of light rays along the curve, and introduces a natural " $2+1$ " splitting of 3-dimensional space. The vector $\xi^{i}$, on the plane orthogonal to the bundle of light rays, is a point of intersection of any of those rays with that plane (see Figure 1). This vector does not depend on $\tau$ and can be defined, as in equation (22), by the relationship

$$
\xi^{i}=P_{j}^{i} x^{j}
$$

where

$$
P_{i j}=\delta_{i j}-k_{i} k_{j}
$$

is the projection operator onto the plane orthogonal to the vector $k^{i}$. The operator has only two algebraically independent components and satisfies the relationship

$$
P_{k}^{i} P_{j}^{k}=P_{j}^{i}
$$

Because of this property we can recast equation (26) into the form

$$
\xi^{i}=P_{j}^{i} \xi^{j}
$$

which shows explicitly that the vector $\xi^{i}$ is constrained to lie in a 2-dimensional plane. Thus, we immediately have for the operation of partial differentiation in this plane

$$
\frac{\partial \xi^{i}}{\partial \xi^{j}}=P_{j}^{i}=P^{i j}=P_{i j}
$$

It is worth noting that the projection operator can be used to raise and lower indices of any geometrical object lying in the plane orthogonal to vector $k^{i}$.

In what follows, it is convenient to consider the spatial components of coordinates $\xi^{i}$ as formally independent with subsequent projection onto the plane when doing differentiation with respect to $\xi^{i}$. Therefore we always use the operator of differentiation with respect to $\xi^{i}$ in combination with the projection operator $P_{j}^{i}$. For example, before the projection we treat

$$
\frac{\partial \xi^{i}}{\partial \xi^{j}}=\delta_{j}^{i}
$$

and for the same expression with subsequent projection

$$
P_{j}^{q} \frac{\partial \xi^{i}}{\partial \xi^{q}}=P_{j}^{i}
$$

which agrees with equations (28) and (30). Moreover, the following rule of differentiation for an arbitrary smooth function $F(t, \mathbf{x})$ holds

$$
\left[\left(\frac{\partial}{\partial x^{i}}+k_{i} \frac{\partial}{\partial t}\right) F(t, \mathbf{x})\right]_{\mathbf{x}=\mathbf{x}_{0}+\mathbf{k}\left(t-t_{0}\right)}=\left(P_{i}^{j} \frac{\partial}{\partial \xi^{j}}+k_{i} \frac{\partial}{\partial \tau}\right) F[\tau, \boldsymbol{\xi}+\mathbf{k} \tau]
$$


Equation (33) is a generalization of the corresponding formula introduced by Kopeikin ( [24], equation (20)) for functions which do not depend explicitly on time $t$. It is worth noting that in the left-hand side of formula (33) one has first to differentiate the function $F(t, \mathbf{x})$ with respect to time $t$ and spatial coordinates $x^{i}$ and, then, to make the substitution $\mathbf{x}=\mathbf{x}_{0}+\mathbf{k}\left(t-t_{0}\right)$. However, one makes corresponding substitutions in the right-hand side of the formula (33) first and only afterwards takes derivatives.

It is useful to stress again that because the coordinates $\xi^{i}$ lie in the plane orthogonal to the vector $k^{i}$ only two of the three $\xi^{1}, \xi^{2}, \xi^{3}$ are, in fact, independent. We also stress that the new variables $\xi^{i}$ and $\tau$ are independent as well. For this reason, the integration of any function, which can be represented as a time derivative with respect to the parameter $\tau$, is always quite straightforward

$$
\int \frac{\partial}{\partial \tau} F(\tau, \boldsymbol{\xi}) d \tau=F(\tau, \boldsymbol{\xi})+C(\boldsymbol{\xi})
$$

where $C(\boldsymbol{\xi})$ is an arbitrary function of the constant impact parameter. Moreover, as the vector $\xi^{i}$ does not depend on time $\tau$, the partial derivatives with respect to $\xi^{i}$ can be removed from within the time integrals when calculating them along the photon's trajectory, that is

$$
\int \frac{\partial}{\partial \xi^{i}} F(\tau, \boldsymbol{\xi}) d \tau=\frac{\partial}{\partial \xi^{i}} \int F(\tau, \boldsymbol{\xi}) d \tau .
$$

Because of these advantages the new independent coordinates $\tau$ and $\xi^{i}$ are quite useful in calculations. The usefulness of the variables $\tau$ and $\xi^{i}$ has been also recognized by Damour \& Esposito-Farèse [23].

The equations of motion of light rays (3) in terms of parameters $\boldsymbol{\xi}$ and $\tau$ are simpler, and after accounting for a freedom in gauge transformations and implementation of relationship (33) assume the form [42]

$$
\begin{aligned}
\ddot{x}^{i}(\tau)= & \frac{1}{2} \hat{\partial}_{i} h_{00}^{c a n .}-\hat{\partial}_{\tau} h_{0 i}^{c a n .}-\frac{1}{2} k^{i} \hat{\partial}_{\tau} h_{00}^{c a n .}-k^{j} \hat{\partial}_{\tau} h_{i j}^{c a n .}+k^{j} \hat{\partial}_{i} h_{0 j}^{c a n .}+ \\
& \frac{1}{2}\left(\hat{\partial}_{i}+k^{i} \hat{\partial}_{\tau}\right) k^{p} k^{q} h_{p q}^{c a n .}-\hat{\partial}_{\tau \tau}\left(w^{i}-k^{i} w^{0}\right)
\end{aligned}
$$

where the following notations are used: $\hat{\partial}_{i} \equiv P_{i j} \partial / \partial \xi^{j}, \hat{\partial}_{\tau} \equiv \partial / \partial \tau$. Let us emphasize once again that the representation of equation (36) is valid in an arbitrary coordinate system and all metric coefficients are taken along the unperturbed trajectory of propagation of the light ray; that is, $h_{\alpha \beta}(t, \mathbf{x})=h_{\alpha \beta}(\tau, \boldsymbol{\xi}+\mathbf{k} \tau)$. We also remark that the right-hand side of equation (36) contains only spatial partial derivatives with the same index " $i$ " as does the left-hand side of the equation. This contrasts with equation (3) where the indices of spatial derivatives are mixed. Equation (36) will be used in sections 5 and 6 for a general treatment of gravitational perturbations of the photon's trajectory and discussion of relativistic time delay and angle of light deflection.

Another useful form of equation (36) may be obtained if one introduces the four-vector $k^{\alpha}=\left(1, k^{i}\right)$. Then we find

$$
\ddot{x}^{i}(\tau)=\frac{1}{2} k^{\alpha} k^{\beta} \hat{\partial}_{i} h_{\alpha \beta}^{c a n} .-\hat{\partial}_{\tau}\left(k^{\alpha} h_{i \alpha}^{c a n .}-\frac{1}{2} k^{i} k^{j} k^{p} q_{j p}^{c a n} .\right)-\hat{\partial}_{\tau \tau}\left(w^{i}-k^{i} w^{0}\right) .
$$

This form of the equation clearly shows that only the first term on the right-hand side contributes to the deflection of light, if observer and source of light are at infinity. Indeed, one integration of (37) with respect to time from $-\infty$ to $+\infty$ brings all first and second time derivatives to zero, due to the asymptotic flatness of the metric tensor. This makes a connection between the formalism of the present paper and that of Damour \& Esposito-Farèse [23] (see also Appendix A).

\section{B. Calculation of Integrals from the Static Part of the Gravitational Field}

The static part of the gravitational field of the deflector contributes to perturbations of light's ray trajectory, defined by the following indefinite integrals [24]

$$
\begin{gathered}
A(\tau, \boldsymbol{\xi}) \equiv \int \frac{d \tau}{r}=\int \frac{d \tau}{\sqrt{d^{2}+\tau^{2}}}=-\ln \left(\sqrt{d^{2}+\tau^{2}}-\tau\right), \\
B(\tau, \boldsymbol{\xi}) \equiv \int A(\tau, \boldsymbol{\xi}) d \tau=-\tau \ln \left(\sqrt{d^{2}+\tau^{2}}-\tau\right)-\sqrt{d^{2}+\tau^{2}},
\end{gathered}
$$


where we have omitted constants of integration which are absorbed by re-definition of constants of integration of unperturbed light trajectory (19). Integrals (38), (39) are formally divergent at the lower limit. However, this divergence is not dangerous for setting the second of the boundary conditions (1) because only derivatives of the integral (38) appear in the result of the first time integration of the equations of motion of light rays, eliminating the divergent part of the integral [43]. With this in mind, it is easy to prove that integrals (38), (39) are in agreement with the boundary conditions (11).

\section{Calculation of Integrals from Time Dependent Part of Gravitational Field}

One meets two ways of calculation of integrals in finding the path of propagation of light in the gravitational field of a localized source emitting gravitational waves. The first method relies upon the use of the Fourier transform (40) and allows one, at least in principle, to calculate all integrals explicitly if one knows the specific structure of the Fourier image of the quadrupole moment of the deflector [44. The advantage of the second method is based on the fact that one deals with the metric depending on retarded time only. This allows one to make a special transformation of variables within the integral which excludes any dependence of the integrands on the impact parameter, and transfers it to the limits of the integrals. Thus, partial derivatives of the integrals can be calculated explicitly without assumptions about the structure of the quadrupole moment of the deflector. Of course, both methods give the same results. However, the second method is more general.

\section{First Method of Integration}

Let us assume the most general aperiodic form for the time variation of the deflector. In linear approximation the total mass and spin of the deflector are conserved quantities [45] so that they do not depend on time at all, and we can consider them as contributing only to the static part of the gravitational field of the deflector [46]. The quadrupole moment is not static. It may be represented through a Fourier transform as

$$
\mathcal{I}_{i j}(t-r)=(2 \pi)^{-1 / 2} \int_{-\infty}^{+\infty} \tilde{\mathcal{I}}_{i j}(\omega) e^{i \omega(t-r)} d \omega
$$

where $\tilde{\mathcal{I}}_{i j}(\omega)$ is the (complex) Fourier image of the quadrupole moment of the deflector which must be specified for any particular source of gravitational waves. Here, we need not know the specific structure of $\tilde{\mathcal{I}}_{i j}(\omega)$ as it will be shown later it is irrelevant for subsequent calculations.

Taking time derivatives of the quadrupole moment yields

$$
\begin{aligned}
& \dot{\mathcal{I}}^{i j}=(2 \pi)^{-1 / 2} \int_{-\infty}^{+\infty}(i \omega) \tilde{\mathcal{I}}_{i j}(\omega) e^{i \omega(t-r)} d \omega, \\
& \ddot{\mathcal{I}}^{i j}=(2 \pi)^{-1 / 2} \int_{-\infty}^{+\infty}\left(-\omega^{2}\right) \tilde{\mathcal{I}}_{i j}(\omega) e^{i \omega(t-r)} d \omega .
\end{aligned}
$$

Generally speaking, arbitrary aperiodic source of gravitational waves have an infinite spectrum. However, it is possible to choose that frequency band which gives the largest contribution to the spectrum. The mean frequency $\Omega$ of this band defines the size of far (wave) zone of the source, as being roughly equal to the wavelength of emitted gravitational waves $\lambda=2 \pi c / \Omega$. For example, if the deflector of light rays is a binary system, then the strongest emission of gravitational waves takes place at twice the mean orbital frequency of the system. For making estimates we can use the following approximations for components of the quadrupole moment

$$
\left|\dot{\mathcal{I}}^{i j}\right| \simeq\left(\mathcal{M} \text { e e c c) } \frac{a}{\lambda}, \quad \quad\left|\ddot{\mathcal{I}}^{i j}\right| \simeq\left(\mathcal{M} e c^{2}\right) \frac{a^{2}}{\lambda^{2}}, \quad \text { etc. },\right.
$$

where $a$ is a characteristic size of the source of gravitational waves and $e$ is its oblateness, quantifying the deviation of the density distribution from spherical symmetry.

When integrating the equations of light propagation using the metric with Fourier transform (40) for the quadrupole moment one meets the following integrals: 


$$
\begin{aligned}
& I_{1}(\tau, \boldsymbol{\xi}, \omega)=\int_{-\infty}^{\tau} \frac{\cos \left[\omega\left(\tau-\sqrt{d^{2}+\tau^{2}}\right)\right]}{\sqrt{d^{2}+\tau^{2}}} d \tau, \\
& I_{2}(\tau, \boldsymbol{\xi}, \omega)=\int_{-\infty}^{\tau} \frac{\sin \left[\omega\left(\tau-\sqrt{d^{2}+\tau^{2}}\right)\right]}{\sqrt{d^{2}+\tau^{2}}} d \tau .
\end{aligned}
$$

In order to evaluate the integrals (44)-(45) it is useful to change the time argument, $\tau$, to the argument $y$, by the transformation

$$
y=\tau-\sqrt{d^{2}+\tau^{2}}
$$

which yields

$$
\tau=\frac{y^{2}-d^{2}}{2 y}, \quad \sqrt{d^{2}+\tau^{2}}=-\frac{1}{2} \frac{d^{2}+y^{2}}{y}, \quad d \tau=\frac{1}{2} \frac{d^{2}+y^{2}}{y^{2}} d y .
$$

While the parameter $\tau$ runs from $-\infty$ to $+\infty$, the new parameter $y$ runs from $-\infty$ to 0 ; that is, $y$ is always negative.

After transforming time arguments, the integrals $I_{1}$ and $I_{2}$ are reduced to the cosine- and sine integrals respectively ( 47], formula 8.230):

$$
\begin{gathered}
I_{1}(\tau, \boldsymbol{\xi}, \omega)=-\mathbf{C i}(\omega y), \\
I_{2}(\tau, \boldsymbol{\xi}, \omega)=-\mathbf{S i}(\omega y),
\end{gathered}
$$

where constants of integration have been omitted. Secondary integration of integrals (48)-(49) along the light trajectory is required as well. Using transformations 46 - (47) we obtain

$$
\begin{aligned}
& J_{1}(\tau, \boldsymbol{\xi}, \omega) \equiv \int_{-\infty}^{\tau} I_{1}(\tau, \boldsymbol{\xi}, \omega) d \tau=-\tau \mathbf{C i}(\omega y)+\frac{1}{2} \omega d^{2}\left[\operatorname{Si}(\omega y)+\frac{\cos (\omega y)}{2 y}\right]+\frac{\sin (\omega y)}{2 \omega}, \\
& J_{2}(\tau, \boldsymbol{\xi}, \omega) \equiv \int_{-\infty}^{\tau} I_{2}(\tau, \boldsymbol{\xi}, \omega) d \tau=-\tau \mathbf{S i}(\omega y)+\frac{1}{2} \omega d^{2}\left[\mathbf{C i}(\omega y)-\frac{\sin (\omega y)}{2 y}\right]+\frac{\cos (\omega y)}{2 \omega},
\end{aligned}
$$

where constants of integration have again been omitted.

Using the Fourier transform of the quadrupole moment (40) and formulae (44), (45), (48), (49) one calculates the important integrals

$$
\begin{gathered}
B_{i j}(\tau, \boldsymbol{\xi}) \equiv \int_{-\infty}^{\tau} \frac{\mathcal{I}_{i j}(t-r)}{r} d t=(2 \pi)^{-1 / 2} \int_{-\infty}^{+\infty} \tilde{\mathcal{I}}_{i j}(\omega) e^{i \omega t^{*}}\left[I_{1}(\tau, \boldsymbol{\xi}, \omega)+i I_{2}(\tau, \boldsymbol{\xi}, \omega)\right] d \omega, \\
C_{i j}(\tau, \boldsymbol{\xi}) \equiv \int_{-\infty}^{\tau} \frac{\dot{\mathcal{I}}_{i j}(t-r)}{r} d t=(2 \pi)^{-1 / 2} \int_{-\infty}^{+\infty} \omega \tilde{\mathcal{I}}_{i j}(\omega) e^{i \omega t^{*}}\left[-I_{2}(\tau, \boldsymbol{\xi}, \omega)+i I_{1}(\tau, \boldsymbol{\xi}, \omega)\right] d \omega, \\
D_{i j}(\tau, \boldsymbol{\xi}) \equiv \int_{-\infty}^{\tau} B_{i j}(\tau, \boldsymbol{\xi}) d t=(2 \pi)^{-1 / 2} \int_{-\infty}^{+\infty} \tilde{\mathcal{I}}_{i j}(\omega) e^{i \omega t^{*}}\left[J_{1}(\tau, \boldsymbol{\xi}, \omega)+i J_{2}(\tau, \boldsymbol{\xi}, \omega)\right] d \omega \\
E_{i j}(\tau, \boldsymbol{\xi}) \equiv \int_{-\infty}^{\tau} C_{i j}(\tau, \boldsymbol{\xi}) d t=(2 \pi)^{-1 / 2} \int_{-\infty}^{+\infty} \omega \tilde{\mathcal{I}}_{i j}(\omega) e^{i \omega t^{*}}\left[J_{2}(\tau, \boldsymbol{\xi}, \omega)-i J_{1}(\tau, \boldsymbol{\xi}, \omega)\right] d \omega
\end{gathered}
$$


where $t^{*}$ is the moment of closest approach of the photon to the origin of coordinate system. In what follows, we need only partial derivatives with respect to the impact parameter of the integrals (52) - (55). These can be calculated rather easily. We have, for example,

$$
\hat{\partial}_{i} I_{1}(\tau, \boldsymbol{\xi}, \omega)=(y r)^{-1} \cos (\omega y) \xi^{i}, \quad \hat{\partial}_{i} I_{2}(\tau, \boldsymbol{\xi}, \omega)=(y r)^{-1} \sin (\omega y) \xi^{i},
$$

and so on. Thus, making use of the inverse Fourier transform we obtain

$$
\begin{aligned}
& \hat{\partial}_{k} B_{i j}(\tau, \boldsymbol{\xi})=(y r)^{-1} \mathcal{I}_{i j}(t-r) \xi^{k}, \\
& \hat{\partial}_{\tau} B_{i j}(\tau, \boldsymbol{\xi})=\left(1-\frac{\tau}{r}\right) \frac{\mathcal{I}_{i j}(t-r)}{y}, \\
& \hat{\partial}_{k} C_{i j}(\tau, \boldsymbol{\xi})=(y r)^{-1} \dot{\mathcal{I}}_{i j}(t-r) \xi^{k} . \\
& \hat{\partial}_{\tau} C_{i j}(\tau, \boldsymbol{\xi})=\left(1-\frac{\tau}{r}\right) \frac{\dot{\mathcal{I}}_{i j}(t-r)}{y},
\end{aligned}
$$

Calculation of partial derivatives from integrals $D_{i j}(\tau, \boldsymbol{\xi})$ and $E_{i j}(\tau, \boldsymbol{\xi})$ may be done without difficulty in a similar fashion using equations (50)-(51).

\section{Second Method of Integration}

The second method also uses the substitutions (46), (47). The integrals (52) - (53) are brought into the form

$$
\begin{aligned}
& B_{i j}(\tau, \boldsymbol{\xi}) \equiv \int_{-\infty}^{\tau} \frac{\mathcal{I}_{i j}(t-r)}{r} d t=-\int_{-\infty}^{y} \frac{\mathcal{I}_{i j}\left(t^{*}+\zeta\right)}{\zeta} d \zeta, \\
& C_{i j}(\tau, \boldsymbol{\xi}) \equiv \int_{-\infty}^{\tau} \frac{\dot{\mathcal{I}}_{i j}(t-r)}{r} d t=-\int_{-\infty}^{y} \frac{\dot{\mathcal{I}}_{i j}\left(t^{*}+\zeta\right)}{\zeta} d \zeta .
\end{aligned}
$$

One sees that the integrands of the integrals do not depend on the parameters $d$ and $\tau$ at all. They are present only in the upper limit of integration. Hence, the integrals (61), (62) are functions of the variable $y$ only, that is $B_{i j}(\tau, \boldsymbol{\xi})=B_{i j}(y)$ and $C_{i j}(\tau, \boldsymbol{\xi})=C_{i j}(y)$. Making use of the transformations (46), (47), the integrals (54), (55) are reduced to the expressions

$$
\begin{aligned}
& D_{i j}(\tau, \boldsymbol{\xi}) \equiv \int_{-\infty}^{\tau} B_{i j}(\tau, \boldsymbol{\xi}) d t=\frac{1}{2} \int_{-\infty}^{y} B_{i j}(\zeta) d \zeta+\frac{d^{2}}{2} \int_{-\infty}^{y} \frac{B_{i j}(\zeta)}{\zeta^{2}} d \zeta, \\
& E_{i j}(\tau, \boldsymbol{\xi}) \equiv \int_{-\infty}^{\tau} C_{i j}(\tau, \boldsymbol{\xi}) d t=\frac{1}{2} \int_{-\infty}^{y} C_{i j}(\zeta) d \zeta+\frac{d^{2}}{2} \int_{-\infty}^{y} \frac{C_{i j}(\zeta)}{\zeta^{2}} d \zeta .
\end{aligned}
$$

Hence, the integrals $D_{i j}(\tau, \boldsymbol{\xi}), E_{i j}(\tau, \boldsymbol{\xi})$ are also functions of the variable $y$ only.

We stress once again that our formalism holds true for arbitrary dependence of the quadrupole moment of the localized source on time, and includes the case of sources which produce bursts of gravitational radiation, such as supernova explosions or coalescence of binary systems, as well as periodic systems. Indeed, suppose that the burst starts at the moment $t_{1}$ and terminates at the moment $t_{2}$. We assume for simplicity that before and after the burst the quadrupole moment of the source is identically zero. During the burst, the tensor function $\mathcal{F}_{i j}(t)$ describes the time 
dependence of the quadrupole moment. Then all formulae derived in this paper hold, if we describe the quadrupole moment of the source as a product of two Heaviside step functions with the tensor function $\mathcal{F}_{i j}(t)$. Thus, for any moment of time we write

$$
\mathcal{I}_{i j}(t)=H\left(t-t_{1}\right) H\left(t_{2}-t\right) \mathcal{F}_{i j}(t),
$$

where the Heaviside step function is defined as follows

$$
H(t-T)= \begin{cases}1 & \text { if } t>T \\ 0 & \text { otherwise. }\end{cases}
$$

Time derivatives of the quadrupole moment are calculated taking into account that $\dot{H}(t-T)=\delta(t-T)$ is the Dirac delta-function, and $\delta\left(t-t_{1}\right) \mathcal{F}_{i j}\left(t_{1}\right)=\delta\left(t-t_{2}\right) \mathcal{F}_{i j}\left(t_{2}\right)=0$. This yields

$$
\dot{\mathcal{I}}_{i j}(t)=H\left(t-t_{1}\right) H\left(t_{2}-t\right) \dot{\mathcal{F}}_{i j}(t), \quad \ddot{\mathcal{I}}_{i j}(t)=H\left(t-t_{1}\right) H\left(t_{2}-t\right) \ddot{\mathcal{F}}_{i j}(t)
$$

and similar formulae for higher derivatives.

It is evident from the structure of integrals (61)-(64) that taking partial derivatives of any of the foregoing integrals is reduced to taking the partial derivative with respect to $y$. In particular, we obtain

$$
\hat{\partial}_{j} B_{p q}(\tau, \boldsymbol{\xi})=-\frac{\mathcal{I}_{p q}\left(t^{*}+y\right)}{y} \hat{\partial}_{j} y=(y r)^{-1} \mathcal{I}_{p q}(t-r) \xi^{j}
$$

which exactly coincides with the result $(57)$ derived above using the inverse Fourier transform method. Second and third partial derivatives of the function $B_{i j}(\tau, \boldsymbol{\xi})$ with respect to the impact parameter will be useful subsequently. They are calculated making use of formula $([68)$. This yields

$$
\hat{\partial}_{j k} B_{p q}(\tau, \boldsymbol{\xi})=(y r)^{-1}\left[P_{j k}+\frac{\xi_{j} \xi_{k}}{y r}-\frac{\xi_{j} \xi_{k}}{r^{2}}\right] \mathcal{I}_{p q}(t-r)-\frac{\xi_{j} \xi_{k}}{y r^{2}} \dot{\mathcal{I}}_{p q}(t-r)
$$

and

$$
\begin{aligned}
\hat{\partial}_{i j k} B_{p q}(\tau, \boldsymbol{\xi})= & (y r)^{-1}\left[\frac{\xi_{i} P_{j k}}{y r}+\frac{2 \xi_{k} P_{i j}}{y r}+\frac{2 \xi_{i} \xi_{j} \xi_{k}}{y^{2} r^{2}}\right. \\
& \left.-\frac{\xi_{i} P_{j k}}{r^{2}}-\frac{2 \xi_{k} P_{i j}}{r^{2}}-\frac{3 \xi_{i} \xi_{j} \xi_{k}}{y r^{3}}+\frac{3 \xi_{i} \xi_{j} \xi_{k}}{r^{4}}\right] \mathcal{I}_{p q}(t-r) \\
& -(y r)^{-1}\left[\frac{\xi_{i} P_{j k}}{r}+\frac{2 \xi_{k} P_{i j}}{r}+\frac{2 \xi_{i} \xi_{j} \xi_{k}}{y r^{2}}-\frac{3 \xi_{i} \xi_{j} \xi_{k}}{r^{3}}\right] \dot{\mathcal{I}}_{p q}(t-r) \\
& +\frac{\xi_{i} \xi_{j} \xi_{k}}{y r^{3}} \ddot{\mathcal{I}}_{p q}(t-r) .
\end{aligned}
$$

We note that the formulae of partial differentiation of $C_{i j}(\tau, \boldsymbol{\xi})$ look the same as for $B_{i j}(\tau, \boldsymbol{\xi})$ after taking into account the fact that the integral (62) depends on the first time derivative of the quadrupole moment. The derivatives of the functionals $E_{i j}(\tau, \boldsymbol{\xi})$ and $D_{i j}(\tau, \boldsymbol{\xi})$ can be obtained using relationships (63)-(64) and derviatives of $B_{i j}(\tau, \boldsymbol{\xi})$ and $C_{i j}(\tau, \boldsymbol{\xi})$. For example,

$$
\begin{aligned}
\hat{\partial}_{j} D_{p q}(\tau, \boldsymbol{\xi}) & =\xi^{j}\left[\frac{B_{p q}(\tau, \boldsymbol{\xi})}{y}+\int_{-\infty}^{y} \frac{B_{i j}(\zeta)}{\zeta^{2}} d \zeta\right] \\
\hat{\partial}_{j k} D_{p q}(\tau, \boldsymbol{\xi}) & =\frac{\xi^{j}}{y} \hat{\partial}_{k} B_{p q}(\tau, \boldsymbol{\xi})+P^{j k}\left[\frac{B_{p q}(\tau, \boldsymbol{\xi})}{y}+\int_{-\infty}^{y} \frac{B_{i j}(\zeta)}{\zeta^{2}} d \zeta\right] \\
\hat{\partial}_{i j k} D_{p q}(\tau, \boldsymbol{\xi}) & =\frac{1}{y}\left[\left(P^{i j}+\frac{\xi^{i} \xi^{j}}{y r}\right) \hat{\partial}_{k} B_{p q}(\tau, \boldsymbol{\xi})+P^{j k} \hat{\partial}_{i} B_{p q}(\tau, \boldsymbol{\xi})+\xi^{j} \hat{\partial}_{i k} B_{p q}(\tau, \boldsymbol{\xi})\right] .
\end{aligned}
$$


It is worth emphasizing that the third partial derivative of $D_{p q}(\tau, \boldsymbol{\xi})$ does not include the integral $B_{p q}(\tau, \boldsymbol{\xi})$ by itself, as might be expected, but only its first and second derivatives. Therefore, the third partial derivative of $D_{p q}(\tau, \boldsymbol{\xi})$ does not depend on the past history of propagation of the light ray (see formulae (68) and (69)).

Now, after making these remarks, we are ready to discuss the relativistic perturbations of the photon's trajectory in the radiative gravitational field of a localized source deflecting light rays.

\section{PERTURBATIONS OF PHOTON'S TRAJECTORY}

We first note that in terms of the new variables $\tau$ and $\xi^{i}$ the components of the "canonical" metric tensor (12)-(14) taken at an arbitrary point on the light ray can be re-written as follows 448]:

$$
\begin{aligned}
h_{00}^{c a n .}(\tau, \boldsymbol{\xi})= & \frac{2 \mathcal{M}}{r}+\left(\hat{\partial}_{i j}+2 k_{i} \hat{\partial}_{j \tau}+k_{i} k_{j} \hat{\partial}_{\tau \tau}\right)\left[\frac{\mathcal{I}_{i j}(t-r)}{r}\right]- \\
& 2\left(k_{i} \hat{\partial}_{j}+k_{i} k_{j} \hat{\partial}_{\tau}\right)\left[\frac{\dot{\mathcal{I}}_{i j}(t-r)}{r}\right]+k_{i} k_{j} \frac{\ddot{\mathcal{I}}_{i j}(t-r)}{r}, \\
h_{0 i}^{c a n .}(\tau, \boldsymbol{\xi})= & -\frac{2 \epsilon_{i p q} \mathcal{S}^{p} x_{N}^{q}}{r^{3}}+2\left(\hat{\partial}_{j}+k_{j} \hat{\partial}_{\tau}\right)\left[\frac{\dot{\mathcal{I}}_{i j}(t-r)}{r}\right]-2 k_{j} \frac{\ddot{\mathcal{I}}_{i j}(t-r)}{r}, \\
h_{i j}^{c a n .}(\tau, \boldsymbol{\xi})= & \delta_{i j} h_{00}^{c a n .}(\tau, \boldsymbol{\xi})+\frac{2}{r} \ddot{\mathcal{I}}_{i j}(t-r),
\end{aligned}
$$

where in the right-hand side of all formulae it is implicitly assumed that variables $t, x^{i}$ are replaced by $\tau$ and $\xi^{i}$, and $\hat{\partial}_{i} \equiv P_{i}^{j} \partial / \partial \xi^{j}, \hat{\partial}_{\tau} \equiv \partial / \partial \tau$. In addition, note that the dot over the quadrupole moment $\mathcal{I}_{i j}$ takes the usual meaning of differentiation with respect to time, which must be completed first, before substitution of $t$ and $x^{i}$ for $\tau$ and $\xi^{i}$, and before taking any other derivative.

The metric tensor (74) - (76) is used in the equations of motion of light rays (36) which are reduced with the help of formula (33) to the expression:

$$
\begin{aligned}
\ddot{x}^{i}(\tau)= & {\left[2 \mathcal{M}\left(\hat{\partial}_{i}-k_{i} \hat{\partial}_{\tau}\right)-2 \mathcal{S}^{p}\left(\epsilon_{i p q} \hat{\partial}_{q \tau}+k_{q} \epsilon_{i p q} \hat{\partial}_{\tau \tau}-k_{j} \epsilon_{j p q} \hat{\partial}_{i q}\right)\right]\left\{\frac{1}{r}\right\}+} \\
& \left(\hat{\partial}_{i p q}-k_{i} \hat{\partial}_{p q \tau}+2 k_{p} \hat{\partial}_{i q \tau}+k_{p} k_{q} \hat{\partial}_{i \tau \tau}-2 k_{i} k_{p} \hat{\partial}_{q \tau \tau}-k_{i} k_{p} k_{q} \hat{\partial}_{\tau \tau \tau}\right)\left\{\frac{\mathcal{I}_{p q}(t-r)}{r}\right\}+ \\
& 2\left(k_{i} k_{p} \hat{\partial}_{q \tau}-\delta_{i p} \hat{\partial}_{q \tau}-\delta_{i p} k_{q} \hat{\partial}_{\tau \tau}+k_{i} k_{p} k_{q} \hat{\partial}_{\tau \tau}\right)\left\{\frac{\dot{\mathcal{I}}_{p q}(t-r)}{r}\right\}-\hat{\partial}_{\tau \tau}\left(w^{i}-k^{i} w^{0}\right),
\end{aligned}
$$

where $w^{i}$ and $w^{0}$ are functions given by relationships (9)-(10). Remarkably, no terms depending on the second time derivatives of the quadrupole moment appear in the equations of motion of light rays (77), because of mutual cancellation. This fact explicitly demonstrates that gravitational waves emitted by localized sources are much more elusive from detection by angular deflection than other authors suggest. It is worth noting that the disappearance of terms with second derivatives from the quadrupole moment is a local phenomena and is not a result of integration of equation (77). This is a characteristic feature of General Relativity. Alternative theories of gravity do not possess such a local cancellation of gravitational wave terms. This cancellation may be used for conducting new tests of General Relativity in the weak, radiative gravitational-field limit.

Let us simplify the equations of motion (77) in order to avoid writing down cumbersome expressions. We introduce two functions $\varphi^{i}$ and $\varphi^{0}$ which generate in (77) the time derivatives of second and higher orders. These functions are defined:

$$
\begin{aligned}
\varphi^{0} & =-2 k_{p} \nabla_{q}\left\{\frac{\mathcal{I}_{p q}(t-r)}{r}\right\}+k_{p} k_{q}\left\{\frac{\dot{\mathcal{I}}_{p q}(t-r)}{r}\right\} \\
\varphi^{i} & =2 \mathcal{S}^{p} k_{q} \epsilon_{i p q}\left\{\frac{1}{r}\right\}-k_{p} k_{q} \nabla_{i}\left\{\frac{\mathcal{I}_{p q}(t-r)}{r}\right\}+2 k_{q}\left\{\frac{\dot{\mathcal{I}}_{i q}(t-r)}{r}\right\}
\end{aligned}
$$


where the differential operator $\nabla_{i} \equiv \partial / \partial x^{i}$ must be applied before the substitution of the unperturbed trajectory of light rays. It can be easily confirmed by straightforward use of formula (33) that the expressions (78)-(79) generate terms with second and third derivatives with respect to $\tau$ in (77). The equations for the path of the light ray now assume the form:

$$
\begin{aligned}
\ddot{x}^{i}(\tau)= & {\left[2 \mathcal{M}\left(\hat{\partial}_{i}-k_{i} \hat{\partial}_{\tau}\right)-2 \mathcal{S}^{p}\left(\epsilon_{i p q} \hat{\partial}_{q \tau}-k_{j} \epsilon_{j p q} \hat{\partial}_{i q}\right)\right]\left\{\frac{1}{r}\right\}+} \\
& \left(\hat{\partial}_{i p q}-k_{i} \hat{\partial}_{p q \tau}+2 k_{p} \hat{\partial}_{i q \tau}\right)\left\{\frac{\mathcal{I}_{p q}(t-r)}{r}\right\}-2 P_{i j} \hat{\partial}_{q \tau}\left\{\frac{\dot{\mathcal{I}}_{j q}(t-r)}{r}\right\}- \\
& \hat{\partial}_{\tau \tau}\left[w^{i}+\varphi^{i}-k^{i}\left(w^{0}+\varphi^{0}\right)\right] .
\end{aligned}
$$

We note that the terms $\varphi^{0}$ and $\varphi^{i}$ are gauge-dependent and can be, in principle, eliminated from the equations of motion (77) by choosing appropriate gauge functions $w^{0}$ and $w^{i}$. However, such a procedure will introduce a reference system with a coordinate grid very sensitive to the direction to a specific source of light rays; that is, to the vector $k^{i}$. The coordinate system obtained in this way will be of trifling practical usage. For this reason we do not recommend the elimination of functions $\varphi^{0}$ and $\varphi^{i}$ from $(77)$ and give preference to the ADM-harmonic coordinate system, which admits a much simpler and unique treatment of observable effects. Thus, we leave the functions $\varphi^{0}$ and $\varphi^{i}$ in the equations of motion of light rays, where gauge functions $w^{0}$ and $w^{i}$ are defined by formulae (B13)-(B14).

Proceeding further in this way and integrating equations (77) one obtains

$$
\begin{aligned}
& \dot{x}^{i}(\tau)=k^{i}+\dot{\Xi}^{i}(\tau) \\
& x^{i}(\tau)=x_{N}^{i}(\tau)+\Xi^{i}(\tau)-\Xi^{i}\left(\tau_{0}\right),
\end{aligned}
$$

where the unperturbed trajectory of light ray $x_{N}^{i}(\tau)$ is determined by the expression (22). The relativistic perturbations to the trajectory are:

$$
\begin{aligned}
\dot{\Xi}^{i}(\tau)= & \left(2 \mathcal{M} \hat{\partial}_{i}+2 \mathcal{S}^{p} k_{j} \epsilon_{j p q} \hat{\partial}_{i q}\right) A(\tau, \boldsymbol{\xi})+\hat{\partial}_{i p q} B_{p q}(\tau, \boldsymbol{\xi})- \\
& \left(2 \mathcal{M} k_{i}+2 \mathcal{S}^{p} \epsilon_{i p q} \hat{\partial}_{q}\right)\left\{\frac{1}{r}\right\}-\left(k_{i} \hat{\partial}_{p q}-2 k_{p} \hat{\partial}_{i q}\right)\left\{\frac{\mathcal{I}_{p q}(t-r)}{r}\right\}-2 P_{i j} \hat{\partial}_{q}\left\{\frac{\dot{\mathcal{I}}_{j q}(t-r)}{r}\right\}- \\
& \hat{\partial}_{\tau}\left[w^{i}+\varphi^{i}-k^{i}\left(w^{0}+\varphi^{0}\right)\right], \\
\Xi^{i}(\tau)= & \left(2 \mathcal{M} \hat{\partial}_{i}+2 \mathcal{S}^{p} k_{j} \epsilon_{j p q} \hat{\partial}_{i q}\right) B(\tau, \boldsymbol{\xi})-\left(2 \mathcal{M} k_{i}-2 \mathcal{S}^{p} \epsilon_{i p q} \hat{\partial}_{q}\right) A(\tau, \boldsymbol{\xi})+ \\
& \hat{\partial}_{i p q} D_{p q}(\tau, \boldsymbol{\xi})-\left(k_{i} \hat{\partial}_{p q}-2 k_{p} \hat{\partial}_{i q}\right) B_{p q}(\tau, \boldsymbol{\xi})-2 P_{i j} \hat{\partial}_{q} C_{j q}(\tau, \boldsymbol{\xi})- \\
& -w^{i}(\tau, \boldsymbol{\xi})-\varphi^{i}(\tau, \boldsymbol{\xi})+k^{i}\left[w^{0}(\tau, \boldsymbol{\xi})+\varphi^{0}(\tau, \boldsymbol{\xi})\right] .
\end{aligned}
$$

We emphasize that before differentiation with respect to time $\tau$ or impact parameter $\xi^{i}$, one has to differentiate the quadrupole moment with respect to time $t$ and make the substitutions: $t \mapsto \tau, r \mapsto \sqrt{d^{2}+\tau^{2}}, r_{0} \mapsto \sqrt{d^{2}+\tau_{0}^{2}}$. We also wish to underline that the only integrals which need be calculated explicitly in expressions (83)- 84) are $A(\tau, \boldsymbol{\xi})$ and $B(\tau, \boldsymbol{\xi})$. All other integrals are acted upon by partial derivatives, which reduce them to ordinary functions as explained in the previous section. This remarkable fact allows considerable simplification of the calculations. This simplification results from the fact that the integrands can be formed from retarded potentials independent of impact parameter, after using the transformation of variables (46). This would be impossible if the metric tensor were not a function of retarded time $t-r$. Thus, retardation simplifies the calculations in the case of time-dependent gravitational 
fields. In the case of a static or stationary gravitational field, the calculation of propagation of light can be done using the same technique since one can always consider a constant multipole also as a (constant) function of retarded time. For this reason, more involved calculations of light propagation (e.g. see [24] and [32]) can be simplified as well.

The functions $w^{i}$ and $w^{0}$, which describe freedom in choosing coordinate systems, are taken from formulae (B13)(B14) of Appendix B. Consequently, the integrals of equations of light propagation (77) expressed in the ADMharmonic coordinate gauge possess a simple interpretation of observable effects, as discussed in the following section.

It is convenient to obtain an expression for unit vector $k^{i}$ written in terms of spatial coordinates of the points of emission, $\mathbf{x}_{\mathbf{0}}$, and observation, $\mathbf{x}$, of the light ray. From formula (82) one has

$$
k^{i}=-K^{i}-\frac{P_{j}^{i}\left[\Xi^{j}(\tau, \boldsymbol{\xi})-\Xi^{j}\left(\tau_{0}, \boldsymbol{\xi}\right)\right]}{\left|\mathbf{x}-\mathbf{x}_{0}\right|},
$$

or more explicitly

$$
\begin{gathered}
k^{i}=-K^{i}-\beta^{i}(\tau, \boldsymbol{\xi})+\beta^{i}\left(\tau_{0}, \boldsymbol{\xi}\right), \\
\beta^{i}(\tau, \boldsymbol{\xi})=\beta_{M}^{i}(\tau, \boldsymbol{\xi})+\beta_{S}^{i}(\tau, \boldsymbol{\xi})+\beta_{Q}^{i}(\tau, \boldsymbol{\xi}),
\end{gathered}
$$

where the relativistic corrections $\beta^{i}(\tau, \boldsymbol{\xi})$ to the vector $K^{i}$ are defined as follows:

$$
\begin{aligned}
\beta_{M}^{i}(\tau, \boldsymbol{\xi}) & =\frac{2 \mathcal{M} \hat{\partial}_{i} B(\tau, \boldsymbol{\xi})}{\left|\mathbf{x}-\mathbf{x}_{0}\right|}, \\
\beta_{S}^{i}(\tau, \boldsymbol{\xi}) & =\frac{2 \mathcal{S}^{p} k_{j} \epsilon_{j p q} \hat{\partial}_{i q} B(\tau, \boldsymbol{\xi})+2 P^{i j} \mathcal{S}^{p} \epsilon_{j p q} \hat{\partial}_{q} A(\tau, \boldsymbol{\xi})}{\left|\mathbf{x}-\mathbf{x}_{0}\right|}, \\
\beta_{Q}^{i}(\tau, \boldsymbol{\xi}) & =\frac{\hat{\partial}_{i p q} D_{p q}(\tau, \boldsymbol{\xi})+2 k_{p} \hat{\partial}_{i q} B_{p q}(\tau, \boldsymbol{\xi})-2 P^{i j} \hat{\partial}_{q} C_{j q}(\tau, \boldsymbol{\xi})-P_{j}^{i}\left[w^{j}(\tau, \boldsymbol{\xi})+\varphi^{j}(\tau, \boldsymbol{\xi})\right]}{\left|\mathbf{x}-\mathbf{x}_{0}\right|} .
\end{aligned}
$$

The relativistic corrections $\beta^{i}\left(\tau_{0}, \boldsymbol{\xi}\right)$ are obtained by replacing the parameter $\tau$ in the numerators of expressions (88)-(90) by $\tau_{0}$. One notes that in equation (86) the unit Euclidean vector

$$
K^{i}=-\frac{x^{i}-x_{0}^{i}}{\left|\mathbf{x}-\mathbf{x}_{0}\right|}
$$

defines the direction from the observer towards the source of light and may be interpreted as a direction in asymptotically flat space-time [49]. Relationship (85) allows us to apply the results of integration of equation of light propagation to the boundary value problem as well. The boundary value problem is formulated in terms of initial $\mathbf{x}_{0}$ and final $\mathbf{x}$ positions of the photon

$$
\mathbf{x}(t)=\mathbf{x}, \quad \mathbf{x}\left(t_{0}\right)=\mathbf{x}_{0},
$$

whilst the initial-boundary value problem (11) is formulated by means of assignment of the initial position $\mathbf{x}_{0}$ and velocity of photon at past null infinity. The relativistic correction to the vector $K^{i}$ contains in its denominator the large numerical value of the distance between observer and source of light. However, the difference $\Xi^{j}(\tau, \boldsymbol{\xi})-\Xi^{j}\left(\tau_{0}, \boldsymbol{\xi}\right)$ in the numerator of (85) may be of the same order as $\left|\mathbf{x}-\mathbf{x}_{0}\right|$ itself. For this reason the relativistic correction in question must be taken into account, in general, for calculation of light deflection in the cases of finite distances of observer or source of light from the localized source of gravitational waves. Only in the case where observer and source of light reside at large distances on opposite sides of the source of gravitational waves, as was assumed in the paper by Damour \& Esposito-Farése (1998), can the relativistic correction $\beta^{i}$ beneglected.

\section{BASIC OBSERVABLE RELATIVISTIC EFFECTS}




\section{A. Time Delay}

The gravitational time delay is derived from equations (82), (84). In order to obtain the expression for the time delay we multiply equation (82) by itself and then find the difference $t-t_{0}$ by taking the square root and using an expansion with respect to small relativistic parameters. This yields:

$$
t-t_{0}=\left|\mathbf{x}-\mathbf{x}_{0}\right|-\mathbf{k} \cdot \boldsymbol{\Xi}(\tau)+\mathbf{k} \cdot \boldsymbol{\Xi}\left(\tau_{0}\right)
$$

or

$$
t-t_{0}=\left|\mathbf{x}-\mathbf{x}_{0}\right|+\Delta_{M}\left(t, t_{0}\right)+\Delta_{S}\left(t, t_{0}\right)+\Delta_{Q}\left(t, t_{0}\right),
$$

where $\left|\mathbf{x}-\mathbf{x}_{0}\right|$ is the usual Euclidean distance [50] between the points of emission, $\mathbf{x}_{0}$, and reception, $\mathbf{x}$, of the photon, $\Delta_{M}$ is the classical Shapiro delay produced by the (constant) spherically symmetric part of the gravitational field of the deflector, $\Delta_{S}$ is the Lense-Thirring or Kerr delay due to the (constant) spin of the localized source of gravitational waves, and $\Delta_{Q}$ describes an additional delay caused by the time dependent quadrupole moment of the source. Specifically we obtain:

$$
\begin{gathered}
\Delta_{M}=2 \mathcal{M} \ln \left[\frac{r+\tau}{r_{0}+\tau_{0}}\right] \\
\Delta_{S}=-2 \epsilon_{i j k} k^{j} \mathcal{S}^{k} \hat{\partial}_{i} \ln \left[\frac{r+\tau}{r_{0}+\tau_{0}}\right] \\
\Delta_{Q}=\hat{\partial}_{i j}\left[B_{i j}(\tau, \boldsymbol{\xi})-B_{i j}\left(\tau_{0}, \boldsymbol{\xi}\right)\right]+\delta_{Q}(\tau, \boldsymbol{\xi})-\delta_{Q}\left(\tau_{0}, \boldsymbol{\xi}\right),
\end{gathered}
$$

where

$$
\begin{aligned}
\delta_{Q}(\tau, \boldsymbol{\xi})= & k^{i}\left(w^{i}+\varphi^{i}\right)-w^{0}-\varphi^{0}= \\
& \frac{1}{2} \hat{\partial}_{\tau}\left[\nabla_{p} \nabla_{q}\left\{\frac{{ }^{(-2)} \mathcal{I}_{p q}(t-r)}{r}\right\}\right]-\nabla_{p} \nabla_{q}\left\{\frac{{ }^{(-1)} \mathcal{I}_{p q}(t-r)}{r}\right\}- \\
& k_{p} k_{q} \hat{\partial}_{\tau}\left\{\frac{\mathcal{I}_{p q}(t-r)}{r}\right\}+2 k_{p} k_{q}\left\{\frac{\dot{\mathcal{I}}_{p q}(t-r)}{r}\right\} .
\end{aligned}
$$

and functions ${ }^{(-1)} \mathcal{I}_{p q}(t-r)$ and ${ }^{(-2)} \mathcal{I}_{p q}(t-r)$ are defined by formula $(\mathrm{B} 5)$ of Appendix B. The expression for the second derivative of function $B_{i j}(\tau, \boldsymbol{\xi})$ has been given in equation (69). The other derivatives appearing in $\Delta_{Q}$ are as follows

$$
\begin{gathered}
\hat{\partial}_{\tau}\left\{\frac{\mathcal{I}_{i j}(t-r)}{r}\right\}=-\frac{y}{r} \frac{\dot{\mathcal{I}}_{i j}(t-r)}{r}-\frac{\tau}{r} \frac{\mathcal{I}_{i j}(t-r)}{r^{2}} \\
\nabla_{p} \nabla_{q}\left\{\frac{{ }^{(-1)} \mathcal{I}_{p q}(t-r)}{r}\right\}=\left[\dot{\mathcal{I}}_{p q}(t-r)+3 \frac{\mathcal{I}_{p q}(t-r)}{r}+3 \frac{(-1) \mathcal{I}_{p q}(t-r)}{r^{2}}\right] \frac{x_{N}^{p} x_{N}^{q}}{r^{3}},
\end{gathered}
$$




$$
\begin{aligned}
\nabla_{p} \nabla_{q}\left\{\frac{{ }^{(-2)} \mathcal{I}_{p q}(t-r)}{r}\right\}= & {\left[\mathcal{I}_{p q}(t-r)+3 \frac{{ }^{(-1)} \mathcal{I}_{p q}(t-r)}{r}+3 \frac{{ }^{(-2)} \mathcal{I}_{p q}(t-r)}{r^{2}}\right] \frac{x_{N}^{p} x_{N}^{q}}{r^{3}}, } \\
\hat{\partial}_{\tau}\left\{\nabla_{p} \nabla_{q} \frac{(-2) \mathcal{I}_{p q}(t-r)}{r}\right\}= & 2\left[\mathcal{I}_{p q}(t-r)+3 \frac{{ }^{(-1)} \mathcal{I}_{p q}(t-r)}{r}+3 \frac{{ }^{(-2)} \mathcal{I}_{p q}(t-r)}{r^{2}}\right] \frac{x_{N}^{q} k^{p}}{r^{3}} \\
& -3 \frac{\tau}{r}\left[\mathcal{I}_{p q}(t-r)+4 \frac{{ }^{(-1)} \mathcal{I}_{p q}(t-r)}{r}+5 \frac{{ }^{(-2)} \mathcal{I}_{p q}(t-r)}{r^{2}}\right] \frac{x_{N}^{p} x_{N}^{q}}{r^{4}} \\
& -\frac{y}{r}\left[\dot{\mathcal{I}}_{p q}(t-r)+3 \frac{\mathcal{I}_{p q}(t-r)}{r}+3 \frac{{ }^{(-1)} \mathcal{I}_{p q}(t-r)}{r^{2}}\right] \frac{x_{N}^{p} x_{N}^{q}}{r^{3}} .
\end{aligned}
$$

The relationship (94) for the time delay has been derived with respect to coordinate time $t$. In order to convert this relationship to observable proper time, we assume for simplicity that the observer is in a state of free fall and that his velocity is negligibly small at the point of observation, with spatial coordinate $\mathbf{x}$. If the observer's velocity is not small an additional Lorentz transformation of time must be applied. Transformation from the ADM-harmonic coordinate time $t$ to proper time $T$ is made with the help of the formula (e.g. see [51])

$$
d T=d t \sqrt{-g_{00}(t, \mathbf{x})}=d t\left(1-\frac{1}{2} h_{00}\right) .
$$

Implementation of formula (B7) for $h_{00}$ and subsequent integration of (103) with respect to time yields

$$
T=\left(1-\frac{\mathcal{M}}{r}\right)\left(t-t_{i}\right)
$$

where $t_{\mathrm{i}}$ is the initial epoch of observation and all velocity-dependent terms are assumed small, as argued above, and are therefore omitted. We also stress that under usual circumstances the distance $r$ is so large that the difference between the observer's proper time and coordinate time can be neglected. Thus, we are allowed to treat coordinate time $t$ as proper time.

We note that the time delay in the propagation of light depends not only on instantaneous functions of retarded time but also on the integrals of time ${ }^{(-1)} \mathcal{I}_{p q}(t-r)$ and ${ }^{(-2)} \mathcal{I}_{p q}(t-r)$. These integrals describe the whole past history of the source of gravitational waves up to the moment of observation. Under usual circumstances, the influence of such integrals on the time delay is expected to be small. However, this question deserves more detailed discussion and will be studied in more detail elsewhere. For example, these terms may be revealed in observations as the "kinematic resonance effect" predicted by Braginsky \& Grishchuk [14]. These terms may be also important for detection of the solar $g$-mode tidal oscillations by the LISA gravitational- wave antenna in space [52].

\section{B. Deflection of Light}

The coordinate direction to the source of light measured at the point of observation $\mathbf{x}$ is defined by the four-vector $p^{\alpha}=\left(1, p^{i}\right)$ where $p^{i}=-\dot{x}^{i}$, or

$$
p^{i}=-k^{i}-\dot{\Xi}^{i},
$$

and the minus sign directs the vector $p^{i}$ from observer to the source of light. However, the coordinate direction $p^{i}$ is not a directly observable quantity. A real observable vector towards the source of light, $s^{\alpha}=\left(1, s^{i}\right)$, is defined with respect to the local inertial frame of the observer. In this frame $s^{i}=-d X^{i} / d T$, where $T$ is the observer's proper time and $X^{i}$ are spatial coordinates of the local inertial frame. We shall assume for simplicity that observer is at rest [53] with respect to the (global) ADM-harmonic coordinate system $\left(t, x^{i}\right)$. Then the infinitesimal transformation from the global ADM-harmonic coordinates $\left(t, x^{i}\right)$ to the local coordinates $\left(T, X^{i}\right)$ is given by the formulas

$$
d T=\Lambda_{0}^{0} d t+\Lambda_{j}^{0} d x^{j} \quad, \quad d X^{i}=\Lambda_{0}^{i} d t+\Lambda_{j}^{i} d x^{j},
$$


where the matrix of transformation $\Lambda_{\beta}^{\alpha}$ is defined by the requirements of orthonormality

$$
g_{\alpha \beta}=\eta_{\mu \nu} \Lambda_{\alpha}^{\mu} \Lambda_{\beta}^{\nu} .
$$

In particular, the orthonormality condition $(\sqrt{107})$ assumes that spatial angles and lengths at the point of observations are measured with the Euclidean metric $\delta_{i j}$. Because the vector $s^{\alpha}$ is isotropic, we conclude that the Euclidean length $|\mathbf{s}|$ of the vector $s^{i}$ is equal to 1 . Indeed, one has

$$
\eta_{\alpha \beta} s^{\alpha} s^{\beta}=-1+\mathbf{s}^{2}=0 .
$$

Hence, $|\mathbf{s}|=1$.

In the linear approximation with respect to $\mathrm{G}$, the matrix of the transformation is as follows [31]

$$
\begin{aligned}
& \Lambda_{0}^{0}=1-\frac{1}{2} h_{00}(t, \mathbf{x}), \\
& \Lambda_{i}^{0}=-h_{0 i}(t, \mathbf{x}), \\
& \Lambda_{0}^{i}=0, \\
& \Lambda_{j}^{i}=\left[1+\frac{1}{2} h_{00}(t, \mathbf{x})\right] \delta_{i j}+\frac{1}{2} h_{i j}^{T T}(t, \mathbf{x}) .
\end{aligned}
$$

Using the transformation (106) we obtain the relationship between the observable vector $s^{i}$ and the coordinate direction $p^{i}$

$$
s^{i}=-\frac{\Lambda_{0}^{i}-\Lambda_{j}^{i} p^{j}}{\Lambda_{0}^{0}-\Lambda_{j}^{0} p^{j}} .
$$

In the linear approximation this takes the form

$$
s^{i}=\left(1+h_{00}-h_{0 j} p^{j}\right) p^{i}+\frac{1}{2} h_{i j}^{T T} p^{j} .
$$

Remembering that vector $|\mathbf{s}|=1$, we find the Euclidean norm of the vector $p^{i}$ from the relationship

$$
|\mathbf{p}|=1-h_{00}+h_{0 j} p^{j}-\frac{1}{2} h_{i j}^{T T} p^{i} p^{j},
$$

which brings equation (111) to the form

$$
s^{i}=m^{i}+\frac{1}{2} P^{i j} m^{q} h_{j q}^{T T}(t, \mathbf{x}),
$$

where the Euclidean unit vector $m^{i}=p^{i} /|\mathbf{p}|$.

Let us now denote by $\alpha^{i}$ the dimensionless vector describing the total angle of deflection of the light ray measured at the point of observation, and calculated with respect to vector $k^{i}$ given at past null infinity. It is defined according to the relationship [32]

$$
\alpha^{i}(\tau, \boldsymbol{\xi})=k^{i}[\mathbf{k} \cdot \dot{\Xi}(\tau, \boldsymbol{\xi})]-\dot{\Xi}^{i}(\tau, \boldsymbol{\xi}),
$$

or

$$
\alpha^{i}(\tau, \boldsymbol{\xi})=-P_{j}^{i} \dot{\Xi}^{j}(\tau, \boldsymbol{\xi}) .
$$

As a consequence of the definitions (105) and (115) we conclude that

$$
m^{i}=-k^{i}+\alpha^{i}(\tau, \boldsymbol{\xi}) .
$$


Accounting for expressions (113), 116), and (86) we obtain for the observed direction to the source of light

$$
s^{i}(\tau, \boldsymbol{\xi})=K^{i}+\alpha^{i}(\tau, \boldsymbol{\xi})+\beta^{i}(\tau, \boldsymbol{\xi})-\beta^{i}\left(\tau_{0}, \boldsymbol{\xi}\right)+\gamma^{i}(\tau, \boldsymbol{\xi}),
$$

where relativistic corrections $\beta^{i}$ are defined by equations (88)-(90) and the perturbation

$$
\gamma^{i}(\tau, \boldsymbol{\xi})=-\frac{1}{2} P^{i j} k^{q} h_{j q}^{T T}(t, \mathbf{x})
$$

If two sources of light (quasars) are observed along the directions $s_{1}^{i}$ and $s_{2}^{i}$ the measured angle $\psi$ between them in the local inertial frame is:

$$
\cos \psi=\mathbf{s}_{1} \cdot \mathbf{s}_{2}
$$

where the dot denotes the usual Euclidean scalar product. It is worth emphasizing that the observed direction to the source of light includes the relativistic deflection of the light ray. This depends not only on quantities at the point of observation but also on $\beta^{i}\left(\tau_{0}, \boldsymbol{\xi}\right)$, at the point of emission of light. This remark reveals that according to relation (119) a single gravitational wave signal may cause different angular displacements for different sources of light located at different distances from the source of gravitational waves.

Without going into further details of the observational procedure we give an explicit expression for the angle $\alpha^{i}$. We have

$$
\alpha^{i}(\tau, \boldsymbol{\xi})=\alpha_{M}^{i}(\tau, \boldsymbol{\xi})+\alpha_{S}^{i}(\tau, \boldsymbol{\xi})+\alpha_{Q}^{i}(\tau, \boldsymbol{\xi})
$$

where

$$
\begin{aligned}
\alpha_{M}^{i}(\tau, \boldsymbol{\xi})= & -2 \mathcal{M} \hat{\partial}_{i} A(\tau, \boldsymbol{\xi}), \\
\alpha_{S}^{i}(\tau, \boldsymbol{\xi})= & -2 \mathcal{S}^{p} k_{j} \epsilon_{j p q} \hat{\partial}_{i q} A(\tau, \boldsymbol{\xi})+2 \mathcal{S}^{p}\left(P^{i j} \epsilon_{j p q} \hat{\partial}_{q}+k_{q} \epsilon_{i p q} \hat{\partial}_{\tau}\right)\left\{\frac{1}{r}\right\} \\
\alpha_{Q}^{i}(\tau, \boldsymbol{\xi})= & -\hat{\partial}_{i p q} B_{p q}(\tau, \boldsymbol{\xi})-P_{i j}\left(2 k_{p} \hat{\partial}_{j q}+k_{p} k_{q} \hat{\partial}_{j \tau}+2 k_{p} \delta_{j q} \hat{\partial}_{\tau \tau}+2 \delta_{j q} \hat{\partial}_{p \tau}\right) \times \\
& \times\left\{\frac{\mathcal{I}_{p q}(t-r)}{r}\right\}+2 P_{i j}\left(\hat{\partial}_{q}+2 k_{q} \hat{\partial}_{\tau}\right)\left\{\frac{\dot{\mathcal{I}}_{j q}(t-r)}{r}\right\}+\frac{1}{2} \hat{\partial}_{i \tau}\left[\nabla_{p} \nabla_{q}\left\{\frac{(-2) \mathcal{I}_{p q}(t-r)}{r}\right\}\right] .
\end{aligned}
$$

The expression for the third spatial derivative of function $B_{p q}(\tau, \xi)$ has been given in equation (70). The other relevant derivatives are:

$$
\begin{aligned}
\hat{\partial}_{j}\left\{\frac{\dot{\mathcal{I}}_{i j}(t-r)}{r}\right\}= & -\xi^{j}\left[\frac{\ddot{\mathcal{I}}_{i j}(t-r)}{r^{2}}+\frac{\dot{\mathcal{I}}_{i j}(t-r)}{r^{3}}\right], \\
\hat{\partial}_{\tau}\left\{\frac{\dot{\mathcal{I}}_{i j}(t-r)}{r}\right\}= & -\frac{y}{r} \frac{\ddot{\mathcal{I}}_{i j}(t-r)}{r}-\frac{\tau}{r} \frac{\dot{\mathcal{I}}_{i j}(t-r)}{r^{2}}, \\
\hat{\partial}_{i q}\left\{\frac{\mathcal{I}_{p q}(t-r)}{r}\right\}= & -P_{i q}\left[\frac{\dot{\mathcal{I}}_{p q}(t-r)}{r^{2}}+\frac{\mathcal{I}_{p q}(t-r)}{r^{3}}\right]+ \\
& \xi_{i} \xi_{q}\left[\frac{\ddot{\mathcal{I}}_{p q}(t-r)}{r^{3}}+\frac{3 \dot{\mathcal{I}}_{p q}(t-r)}{r^{4}}+\frac{3 \mathcal{I}_{p q}(t-r)}{r^{5}}\right],
\end{aligned}
$$




$$
\begin{aligned}
\hat{\partial}_{i \tau}\left\{\frac{\mathcal{I}_{p q}(t-r)}{r}\right\}= & \frac{y}{r}\left[\frac{\ddot{\mathcal{I}}_{p q}(t-r)}{r^{2}}+\frac{\dot{\mathcal{I}}_{p q}(t-r)}{r^{3}}\right] \xi_{i}+ \\
& \frac{\tau}{r}\left[2 \frac{\dot{\mathcal{I}}_{p q}(t-r)}{r^{3}}+3 \frac{\mathcal{I}_{p q}(t-r)}{r^{4}}\right] \xi_{i} \\
\hat{\partial}_{\tau \tau}\left\{\frac{\mathcal{I}_{p q}(t-r)}{r}\right\}= & \frac{y^{2}}{r^{2}} \frac{\ddot{\mathcal{I}}_{p q}(t-r)}{r}+\left(\frac{2 y \tau}{r^{2}}-1\right) \frac{\dot{\mathcal{I}}_{p q}(t-r)}{r^{2}}+\left(\frac{3 \tau^{2}}{r^{2}}-1\right) \frac{\mathcal{I}_{p q}(t-r)}{r^{3}} .
\end{aligned}
$$

Straightforward but tedious calculation of the last term in equation (123) yields

$$
\begin{aligned}
\hat{\partial}_{i}\left\{\nabla_{p} \nabla_{q} \frac{{ }^{(-2)} \mathcal{I}_{p q}(t-r)}{r}\right\}=2\left[\mathcal{I}_{p q}(t-r)+3 \frac{{ }^{(-1)} \mathcal{I}_{p q}(t-r)}{r}+3 \frac{{ }^{(-2)} \mathcal{I}_{p q}(t-r)}{r^{2}}\right] \frac{x_{N}^{p} P_{i q}}{r^{3}} \\
-\left[\dot{\mathcal{I}}_{p q}(t-r)+6 \frac{\mathcal{I}_{p q}(t-r)}{r}+15 \frac{{ }^{(-1)} \mathcal{I}_{p q}(t-r)}{r^{2}}+15 \frac{{ }^{(-2)} \mathcal{I}_{p q}(t-r)}{r^{3}}\right] \frac{x_{N}^{p} x_{N}^{q} \xi^{i}}{r^{4}}
\end{aligned}
$$

and

$$
\begin{aligned}
& \hat{\partial}_{i \tau}\left\{\nabla_{p} \nabla_{q} \frac{{ }^{(-2)} \mathcal{I}_{p q}(t-r)}{r}\right\}=2 {\left[\mathcal{I}_{p q}(t-r)+3 \frac{{ }^{(-1)} \mathcal{I}_{p q}(t-r)}{r}+3 \frac{{ }^{(-2)} \mathcal{I}_{p q}(t-r)}{r^{2}}\right] \frac{k^{p} P_{i q}}{r^{3}} } \\
&-6 \frac{\tau}{r}\left[\mathcal{I}_{p q}(t-r)+4 \frac{(-1) \mathcal{I}_{p q}(t-r)}{r}+5 \frac{{ }^{(-2)} \mathcal{I}_{p q}(t-r)}{r^{2}}\right] \frac{P_{i q} x_{N}^{p}}{r^{4}}-2 \frac{y}{r}\left[\dot{\mathcal{I}}_{p q}(t-r)+3 \frac{\mathcal{I}_{p q}(t-r)}{r}+3 \frac{{ }^{(-1)} \mathcal{I}_{p q}(t-r)}{r^{2}}\right] \frac{k^{p} P_{i q}}{r^{3}} \\
&-2\left[\dot{\mathcal{I}}_{p q}(t-r)+\right.\left.6 \frac{\mathcal{I}_{p q}(t-r)}{r}+15 \frac{{ }^{(-1)} \mathcal{I}_{p q}(t-r)}{r^{2}}+15 \frac{{ }^{(-2)} \mathcal{I}_{p q}(t-r)}{r^{3}}\right] \frac{\xi^{i} k^{p} x_{N}^{q}}{r^{4}} \\
&+4 \frac{\tau}{r}\left[\dot{\mathcal{I}}_{p q}(t-r)+\frac{15}{2} \frac{\mathcal{I}_{p q}(t-r)}{r}+\frac{45}{2} \frac{(-1) \mathcal{I}_{p q}(t-r)}{r^{2}}+\frac{45}{2} \frac{{ }^{(-2)} \mathcal{I}_{p q}(t-r)}{r^{3}}\right] \frac{\xi^{i} x_{N}^{p} x_{N}^{q}}{r^{5}} \\
&+\frac{y}{r}\left[\ddot{\mathcal{I}}_{p q}(t-r)+6 \frac{\dot{\mathcal{I}}_{p q}(t-r)}{r}+15 \frac{\mathcal{I}_{p q}(t-r)}{r^{2}}+15 \frac{(-1) \mathcal{I}_{p q}(t-r)}{r^{3}}\right] \frac{\xi^{i} x_{N}^{p} x_{N}^{q}}{r^{4}}
\end{aligned}
$$

We note that the angular displacement in astrometric positions of sources of light in the sky depends not only on quantities that are instantaneous functions of retarded time, but also on integrals over time ${ }^{(-1)} \mathcal{I}_{p q}(t-r)$ and ${ }^{(-2)} \mathcal{I}_{p q}(t-r)$, which describe the whole past history of the source of gravitational waves up to the moment of observation. Under usual circumstances the influence of such integrals on the deflection of light is expected to be small. However, this question deserves more detailed discussion and will be discussed elsewhere.

\section{DISCUSSION}

It is remarkable that among all the integrals required for calculation of the trajectory of the light ray, only $B_{i j}(\tau, \boldsymbol{\xi})$ enters the expressions (97), (123) for time delay and deflection angle. Furthermore, it is remarkable that we need not know this integral explicitly, but only its second and third derivatives with respect to impact parameter. These are given in equations (69) and (70). With the knowledge of these derivatives, and derivatives of other functions given in 
the previous section, we have complete information about the functional structure of the relativistic time delay and the angle of light deflection produced by any localized gravitating system possessing a time-dependent quadrupole moment $\mathcal{I}_{i j}(t)$.

This structure indicates that the explicit time dependence of the quadrupole moment completely determines the results of astrometric and timing observations. We shall not consider this problem in the present paper, leaving it for future exploration.

Our concern in this section is the simplification of the general formalism developed in the foregoing text. In order to do this we consider three limiting cases:

1. The impact parameter $d$ is much smaller than the distance from the localized source of gravitational waves to both the observer, $r$, and to the source of light, $r_{0}$. The source of light is behind the source of gravitational waves (see Figure 2);

2. The impact parameter $d$ is much smaller than the distance from the localized source of gravitational waves to the observer, $r$, and to the source of light, $r_{0}$. The source of light is on the same side of the source of gravitational waves as the observer (see Figure 3);

3. The distance $R$ from the source of light rays to the observer is much smaller than distances from the observer or from the source of light to the localized source of gravitational waves. The impact parameter $d$ may be comparable with the distance from the deflector to observer or the source of light (see Figure (1)).

We will conventionally refer to the cases 1 and 2 as those of small impact parameter, with numerical values of $\tau_{0}<0$ and $\tau_{0}>0$ respectively. Case 3 is that of large impact parameter, and its small numerical values are covered by the formalism as well, as will be clear in section 7.3 below.

\section{A. Case 1. Small Impact Parameter $\left(\tau_{0}<0\right)$}

1. Asymptotic expansions of independent variables

We shall assume in this section that the condition $d \ll \min \left[r, r_{0}\right]$ holds. Let $L=\min \left[r, r_{0}\right]$ and recall that $\tau=\sqrt{r^{2}-d^{2}}$ and $\tau_{0}=-\sqrt{r_{0}^{2}-d^{2}}<0$ (see Figure 2). This yields

$$
y=\sqrt{r^{2}-d^{2}}-r=-\frac{d^{2}}{2 r}-\frac{d^{4}}{8 r^{3}}+\ldots
$$

and

$$
y_{0}=-\sqrt{r_{0}^{2}-d^{2}}-r_{0}=-2 r_{0}+\frac{d^{2}}{2 r_{0}}+\frac{d^{4}}{8 r_{0}^{3}}+\ldots,
$$

where dots denote terms of higher order, $r$ is the constant distance from the deflector to observer, and $r_{0}$ is the constant distance from the deflector to the point of emission of light. Using these expansions we find:

$$
t=t^{*}+r-\frac{d^{2}}{2 r}+\ldots, \quad t_{0}=t^{*}-r_{0}+\frac{d^{2}}{2 r_{0}}+\ldots
$$

These can be used for Taylor expansion of functions about the time $t^{*}$, the moment of the closest approach of light ray to the deflector. Specifically, if we assume convergence of this Taylor series we find:

$$
\begin{gathered}
\mathcal{I}_{i j}(t-r)=\mathcal{I}_{i j}\left(t^{*}\right)-\frac{d^{2}}{2 r} \dot{\mathcal{I}}_{i j}\left(t^{*}\right)+\ldots, \\
\mathcal{I}_{i j}\left(t_{0}-r_{0}\right)=\mathcal{I}_{i j}\left(t^{*}-2 r_{0}\right)+\frac{d^{2}}{2 r_{0}} \dot{\mathcal{I}}_{i j}\left(t^{*}-2 r_{0}\right)+\ldots,
\end{gathered}
$$


where dots again denote terms of higher order. Convergence of the time series given above requires:

$$
\frac{\omega d^{2}}{c r} \ll 1, \quad \text { and } \quad \frac{\omega d^{2}}{c r_{0}} \ll 1,
$$

where $\omega$ is the highest frequency of gravitational waves emitted by the deflector. If the source of light rays and observer are at infinite distances from the deflector then the requirements (136) are satisfied automatically, irrespective of the structure of the Fourier spectrum of the quadrupole moment of the deflector. In practical situations such an assumption may not be always satisfied. For this reason, it will be more natural to avoid the Taylor expansions of the quadrupole moment with respect to retarded time. It is also worth noting that in the case of small impact parameter we have

$$
(y r)^{-1}=-\frac{2}{d^{2}}+\frac{1}{2 r^{2}}+\frac{d^{2}}{8 r^{4}}+\ldots
$$

and

$$
\left(y_{0} r_{0}\right)^{-1}=-\frac{1}{2 r_{0}^{2}}-\frac{d^{2}}{8 r_{0}^{4}}+\ldots
$$

The foregoing expansions then yield

$$
\begin{aligned}
\hat{\partial}_{j} B_{p q}(\tau, \boldsymbol{\xi}) & =\left(-2 \hat{\partial}_{j} \ln d+\frac{\xi^{j}}{2 r^{2}}\right) \mathcal{I}_{j k}(t-r)+\ldots, \\
\hat{\partial}_{j} B_{p q}\left(\tau_{0}, \boldsymbol{\xi}\right) & =-\frac{\xi^{j}}{2 r_{0}^{2}} \mathcal{I}_{j k}\left(t_{0}-r_{0}\right)+\ldots \\
\hat{\partial}_{j k} B_{p q}(\tau, \boldsymbol{\xi}) & =-2 \hat{\partial}_{j k} \ln d \mathcal{I}_{p q}(t-r)+\frac{2}{r} n_{j} n_{k} \dot{\mathcal{I}}_{p q}(t-r)+\ldots, \\
\hat{\partial}_{j k} B_{p q}\left(\tau_{0}, \boldsymbol{\xi}\right) & =-\frac{1}{2 r_{0}^{2}} P_{j k} \mathcal{I}_{p q}\left(t_{0}-r_{0}\right)+\ldots, \\
\hat{\partial}_{i j k} B_{p q}(\tau, \boldsymbol{\xi}) & =-2\left[\mathcal{I}_{p q}(t-r)+\frac{d^{2}}{2 r} \dot{\mathcal{I}}_{p q}(t-r)\right] \hat{\partial}_{i j k} \ln d+\ldots, \\
\hat{\partial}_{i j k} B_{p q}\left(\tau_{0}, \boldsymbol{\xi}\right) & =O\left(\frac{1}{r_{0}^{3}}\right)
\end{aligned}
$$

and

$$
\begin{aligned}
\hat{\partial}_{i j k} D_{p q}(\tau, \boldsymbol{\xi}) & =-2 r \mathcal{I}_{p q}(t-r) \hat{\partial}_{i j k} \ln d-\frac{4 n^{i} n^{j} n^{k}}{d} \dot{\mathcal{I}}_{p q}(t-r)+\ldots \\
\hat{\partial}_{i j k} D_{p q}\left(\tau_{0}, \boldsymbol{\xi}\right) & =O\left(\frac{1}{r_{0}^{3}}\right)
\end{aligned}
$$

In addition we have

$$
\begin{aligned}
\delta_{Q}(\tau, \boldsymbol{\xi}) & =\frac{1}{r} k^{p} k^{q} \dot{\mathcal{I}}_{p q}(t-r)+\ldots, \\
\delta_{Q}\left(\tau_{0}, \boldsymbol{\xi}\right) & =O\left(\frac{1}{r_{0}^{2}}\right) .
\end{aligned}
$$

We note that the leading terms of the expansions decay much faster (at least as $1 / r_{0}^{2}$ ) at the point of emission of light than those at the point of observation. This indicates that the main contribution to the effects of time delay and 
deflection of light arise along the path of the light ray from the localized source of gravitational waves to the observer. We discuss this question in more detail in the following section.

The asymptotic expansions of integrals (38) - (39) describing propagation of light rays in the static part of gravitational field of the deflector are:

$$
\begin{gathered}
A(\tau, \boldsymbol{\xi})=-2 \ln d+\ln 2 r-\frac{d^{2}}{4 r^{2}}+\ldots \\
A\left(\tau_{0}, \boldsymbol{\xi}\right)=-\ln 2 r_{0}+\frac{d^{2}}{4 r_{0}^{2}}+\ldots \\
B(\tau, \boldsymbol{\xi})=-r-2 r \ln d+r \ln 2 r-\frac{d^{2}}{2 r}\left[\frac{1}{2}-\ln \left(\frac{d^{2}}{2 r}\right)\right] \ldots, \\
B\left(\tau_{0}, \boldsymbol{\xi}\right)=-r_{0}+r_{0} \ln 2 r_{0}-\frac{d^{2}}{2 r_{0}}\left(\frac{1}{2}+\ln 2 r_{0}\right)+\ldots
\end{gathered}
$$

These expansions are used for calculation of asymptotic expressions for time delay and the angle of deflection of light rays.

\section{Asymptotic expressions for time delay and the angle of light deflection}

The static part of time delay and deflection angle are:

$$
\begin{aligned}
\Delta_{M} & =-4 \mathcal{M} \ln d+2 \mathcal{M} \ln \left(4 r r_{0}\right)+\ldots, \\
\Delta_{S} & =-4 \epsilon_{j i p} k^{j} \mathcal{S}^{p} \hat{\partial}_{i}\left[\ln d-\frac{1}{2} \ln \left(4 r r_{0}\right)\right]+\ldots, \\
\alpha_{M}^{i}(\tau, \boldsymbol{\xi}) & =4 \mathcal{M} \hat{\partial}_{i}\left[\ln d-\frac{1}{2} \ln \left(4 r r_{0}\right)\right]+\ldots, \\
\alpha_{S}^{i}(\tau, \boldsymbol{\xi}) & =4 \epsilon_{j p q} k^{p} \mathcal{S}^{q} \hat{\partial}_{i j}\left[\ln d-\frac{1}{2} \ln \left(4 r r_{0}\right)\right]+\ldots, \\
\beta_{M}^{i}(\tau, \boldsymbol{\xi}) & =-\frac{r}{R} \alpha_{M}^{i}(\tau, \boldsymbol{\xi})+\ldots, \\
\beta_{S}^{i}(\tau, \boldsymbol{\xi}) & =-\frac{r}{R} \alpha_{S}^{i}(\tau, \boldsymbol{\xi})-\frac{4}{R} P^{i j} \mathcal{S}^{k} \epsilon_{j k q} \hat{\partial}_{q} \ln d+\ldots,
\end{aligned}
$$

where we have neglected the angle $\gamma^{i}(\tau, \boldsymbol{\xi})$ because it is small (recall that $\left.\gamma^{i}(\tau, \boldsymbol{\xi}) \simeq P^{i j} k^{q} h_{j q}^{T T}\right)$.

Asymptotic expressions for the time delay and angle of deflection caused by the quadrupole moment are:

$$
\Delta_{Q}=-2 \mathcal{I}_{i j}(t-r) \hat{\partial}_{i j} \ln d+\frac{1}{r}\left(2 n_{i} n_{j}+k_{i} k_{j}\right) \dot{\mathcal{I}}_{i j}(t-r)+\ldots,
$$

and 


$$
\begin{aligned}
& \alpha_{Q}^{i}(\tau, \boldsymbol{\xi})=2\left[\mathcal{I}_{j k}(t-r)+\frac{d^{2}}{2 r} \dot{\mathcal{I}}_{j k}(t-r)\right] \hat{\partial}_{i j k} \ln d+\ldots \\
& \beta_{Q}^{i}(\tau, \boldsymbol{\xi})=-\frac{r}{R} \alpha_{Q}^{i}(\tau, \boldsymbol{\xi})-\frac{4}{R}\left[k^{j} \mathcal{I}_{j k}(t-r) \hat{\partial}_{i k} \ln d+\frac{1}{2} \xi^{i} \dot{\mathcal{I}}_{j k}(t-r) \hat{\partial}_{j k} \ln d\right]+\ldots,
\end{aligned}
$$

where $n^{i}=\xi^{i} / d$ is the unit vector directed along the impact parameter, $R=\left|\mathbf{x}-\mathbf{x}_{0}\right|$, and dots denote terms of higher order [54]. The angle $\beta^{i}\left(\tau_{0}, \boldsymbol{\xi}\right)$ at the point of emission of light is negligibly small and, for this reason, its exact expression has been not shown.

Our calculations show that the time dependent part of the time delay and light deflection by the quadrupole moment of a localized source of gravitational field fall off in the first approximation as the inverse square and inverse cube of the impact parameter $d$ respectively. For this reason there is no magnification of the gravitational wave signal in astrometric or pulsar timing observations as some authors have suggested [19] - 21. In particular, terms proportional to $1 / d$, or even to $1 / d^{2}$, appear only in terms of high order in the expansion (160) and are always multiplied by the factor $1 / r$ to some power.

The first term of formula (159) was first derived by Sazhin [7] for the special case of a binary system with a specific orientation of its orbital plane. Our derivation of formula (160) improves and gives independent confirmation of the result established previously by Damour \& Esposito-Farèse [23] using another mathematical technique based on application of Fourier transform and pure harmonic coordinates. For completeness we have repeated the calculations of Damour \& Esposito-Farèse 23] for the effect of deflection of light rays by localized sources of gravitational waves in ADM rather than harmonic coordinates (see Appendix A). The result coincides completely with that of Damour \& Esposito-Farèse 23 and clearly demonstrates the gauge invariance of the result. However, our technique is more general and powerful. Our formalism is valid for any relative position of observer, source of light, and source of gravitational waves, and with finite or infinite separations. The method developed by Damour \& Esposito-Farèse [23] is valid only for infinite separations and for small values of impact parameter. In particular, we note that while Damour \& Esposito-Farèse [23] find that the deflection depends on the time $t^{*}$ of the closest approach of light to the deflector, our calculation shows that it depends on the retarded time $t-r$. This difference is insignificant for extremely large separation of the light source and observer from the deflector, and small impact parameter, but it can be important in the cases of finite distances or large impact parameter.

It is important to realize that in the case of a small impact parameter, the basic time-dependent contribution to the bending of light and time delay by the gravitational field of a localized source of gravitational waves comes from the static part of the near-zone gravitational field of the source taken at the retarded time (cf. formulae (50)-(53) from [24]). In this respect it is worth emphasizing that the formula for the bending of light given in paper [23] as well as in Appendix A is valid under two assumptions: 1) the impact parameter $d$ is small compared with the distance to the observer $r, 2$ ) the velocity of matter inside the source of gravitational radiation is much smaller than the speed of light (the slow-motion approximation).

The first assumption is rather trivial, since the impact parameter $d$ is the only finite distance when the source of light and observer are at infinity. The second assumption appears because paper [23] uses the Taylor expansion of the Fourier image of the tensor of energy-momentum of matter with respect to wave vector $\mathbf{k}$ (see equations (3.3) and (3.4) of paper [23]). This expansion is mathematically equivalent to the use of a slow-motion approximation [55] which, in particular, restricts the nature of the source of gravitational waves so that its Fourier spectrum is not allowed to include too high frequencies.

In contrast, the general formalism given in the present paper produces results (159) and (160) applicable to arbitrary sources of gravitational waves, including gravitational radiation bursts with internal velocity of matter comparable to the speed of light [56]. Moreover, we do not assume positions of observer and the source of light to be at infinity.

If we introduce the notion of the transverse-traceless (TT) and longitudinal (L) tensors [24], [33] with respect to the direction of propagation of light rays

$$
\begin{aligned}
\mathcal{I}_{i j}^{T T} & =\mathcal{I}_{i j}+\frac{1}{2}\left(\delta_{i j}+k_{i} k_{j}\right) k_{p} k_{q} \mathcal{I}_{p q}-\left(\delta_{i p} k_{j} k_{q}+\delta_{j p} k_{i} k_{q}\right) \mathcal{I}_{p q}, \\
\mathcal{I}_{i j}^{L} & =k_{i} k_{p} \mathcal{I}_{j p}+k_{j} k_{p} \mathcal{I}_{i p}-k_{i} k_{j}\left(k_{p} k_{q} \mathcal{I}_{p q}\right)
\end{aligned}
$$

the expressions (159)-(160) are reduced to the form

$$
\Delta_{Q}=-2 \mathcal{I}_{i j}^{T T}(t-r) \hat{\partial}_{i j} \ln d+\frac{2}{r} n^{i} n^{j} \dot{\mathcal{I}}_{i j}^{T T}(t-r)+\ldots,
$$




$$
\begin{aligned}
& \alpha_{Q}^{i}(\tau, \boldsymbol{\xi})=2\left[\mathcal{I}_{j k}^{T T}(t-r)+\frac{d^{2}}{2 r} \dot{\mathcal{I}}_{j k}^{T T}(t-r)\right] \hat{\partial}_{i j k} \ln d+\ldots \\
& \beta_{Q}^{i}(\tau, \boldsymbol{\xi})=-\frac{r}{R} \alpha_{Q}^{i}(\tau, \boldsymbol{\xi})-\frac{4}{R}\left[k^{j} \mathcal{I}_{j k}^{L}(t-r) \hat{\partial}_{i k} \ln d+\frac{1}{2} \xi^{i} \dot{\mathcal{I}}_{j k}^{T T}(t-r) \hat{\partial}_{j k} \ln d\right]+\ldots
\end{aligned}
$$

which reveals explicitly that only the transverse-traceless part of the quadrupole moment of the localized source of gravitational waves contributes to the leading terms. However, terms of higher order can depend on the longitudinal part of the quadrupole moment as well.

It is interesting to see that if we apply the expansions (134)-(135), use the approximation of a gravitational lens, and omit all terms depending on time derivatives of the quadrupole moment, the expressions for the time delay and the angle of light deflection can be reduced to the formulae [57]

$$
t-t_{0}=\left|\mathbf{x}-\mathbf{x}_{0}\right|-4 \psi+2 \mathcal{M} \ln \left(4 r r_{0}\right), \quad \alpha_{i}=4 \hat{\partial}_{i} \psi,
$$

where $\psi$ is the gravitational lens potential [58] having the form

$$
\psi=\left[\mathcal{M}+\epsilon_{j p q} k^{p} \mathcal{S}^{q} \hat{\partial}_{j}+\frac{1}{2} \mathcal{I}_{p q}^{T T}\left(t^{*}\right) \hat{\partial}_{p q}\right] \ln d .
$$

Scrutiny of the multipole structure of $\psi$ in cosmological gravitational lenses may reveal the presence of dark matter in the lens and identify the position of its center of mass, velocity and density distribution.

Expression (168) includes explicit dependence on mass, spin, and quadrupole moment of the deflector and generalizes that given by Damour \& Esposito-Farèse [23] by accounting for the spin multipole. A similar result for the gravitational lens potential was obtained independently by Kopeikin [24] in the case of a stationary gravitational field for the deflector. The fact that the deflection angle can be represented as a gradient of the gravitational lens potential $\psi$ explicitly indicates that the, so-called, frame-dragging effect in gravitational lenses [59] can give a noticeable contribution to the deflection angle. Frame-dragging also produces a small displacement of the image of the background source from the plane formed by the two vectors directed from the observer toward the image of the light source and toward the gravitational lens. This torsional displacement of the image is produced only by the component of spin of the deflector directed along the light ray (see the second term in equation (158). The overall effect of the torsion is of order $d / r$ smaller than the main terms in the expression (168). These remarks dispel a seemingly common opinion that rotation of the deflector prevents representation of the deflection angle as a gradient of a gravitational lens potential. Similar conclusions can be derived from [24] and [32]. Ibáñez \& Martin [60] and Ibáñez 61] give a formula for effects of frame-dragging equivalent to the spin-dependent term in (168), although they do not calculate all necessary integrals or estimate residual terms.

Taking into account formula (117) and expressions for $\alpha^{i}, \beta^{i}$, and $\gamma$ we obtain the vector equation for a gravitational lens

$$
s^{i}=K^{i}+\frac{r_{0}}{R} \alpha^{i},
$$

where $\alpha^{i}$ is given by relationships (167), (168) and we have taken into account that in the case under consideration $R \simeq r+r_{0}$. One recognizes that when distances are finite the deflection angle with respect to vector $K^{i}$ is not simply $\alpha^{i}$ but the product of $r_{0} / R$ and $\alpha^{i}$. In the limit when $K^{i} \rightarrow k^{i}$, which is equaivalent to $\beta^{i} \rightarrow 0$, or $r=$ const., $r_{0} \rightarrow \infty$ the observed angle of deflection approaches the total angle of deflection $\alpha^{i}$, as it must in this limiting case.

\section{B. Case 2. Small Impact Parameter $\left(\tau_{0}>0\right)$}

\section{Asymptotic expansions of independent variables}

We shall again assume in this section that the condition $d \ll \min \left[r, r_{0}\right]$ holds and that $\tau=\sqrt{r^{2}-d^{2}}$ and $\tau_{0}=$ $\sqrt{r_{0}^{2}-d^{2}}>0$ (see Figure 3). This yields 


$$
y=\sqrt{r^{2}-d^{2}}-r=-\frac{d^{2}}{2 r}-\frac{d^{4}}{8 r^{3}}+\ldots
$$

and

$$
y_{0}=\sqrt{r_{0}^{2}-d^{2}}-r_{0}=-\frac{d^{2}}{2 r_{0}}-\frac{d^{4}}{8 r_{0}^{3}}+\ldots,
$$

where dots denote terms of higher order, $r$ is the constant distance from the deflector to observer, and $r_{0}$ is the constant distance from the deflector to the point of emission of light. Using these expansions we obtain the following decompositions

$$
t=t^{*}+r-\frac{d^{2}}{2 r}+\ldots, \quad t_{0}=t^{*}+r_{0}-\frac{d^{2}}{2 r_{0}}+\ldots .
$$

These can be used for Taylor expansion of functions that depend on retarded time about the time $t^{*}$. In this case $t^{*}$ is the moment of closest approach of the light ray trajectory extrapolated backward to the deflector (see Figure 3 ). If we assume convergence of this Taylor series we find:

$$
\begin{gathered}
\mathcal{I}_{i j}(t-r)=\mathcal{I}_{i j}\left(t^{*}\right)-\frac{d^{2}}{2 r} \dot{\mathcal{I}}_{i j}\left(t^{*}\right)+\ldots, \\
\mathcal{I}_{i j}\left(t_{0}-r_{0}\right)=\mathcal{I}_{i j}\left(t^{*}\right)-\frac{d^{2}}{2 r_{0}} \dot{\mathcal{I}}_{i j}\left(t^{*}\right)+\ldots,
\end{gathered}
$$

where dots again denote terms of higher order. We also have

$$
(y r)^{-1}=-\frac{2}{d^{2}}+\frac{1}{2 r^{2}}+\frac{d^{2}}{8 r^{4}}+\ldots
$$

and

$$
\left(y_{0} r_{0}\right)^{-1}=-\frac{2}{d^{2}}+\frac{1}{2 r_{0}^{2}}+\frac{d^{2}}{8 r_{0}^{4}}+\ldots
$$

The foregoing expansions yield

$$
\begin{aligned}
\hat{\partial}_{j} B_{p q}(\tau, \boldsymbol{\xi}) & =\left(-2 \hat{\partial}_{j} \ln d+\frac{\xi^{j}}{2 r^{2}}\right) \mathcal{I}_{j k}(t-r)+\ldots, \\
\hat{\partial}_{j} B_{p q}\left(\tau_{0}, \boldsymbol{\xi}\right) & =\left(-2 \hat{\partial}_{j} \ln d+\frac{\xi^{j}}{2 r_{0}^{2}}\right) \mathcal{I}_{j k}\left(t_{0}-r_{0}\right)+\ldots \\
\hat{\partial}_{j k} B_{j k}(\tau, \boldsymbol{\xi}) & =-2 \hat{\partial}_{j k} \ln d \mathcal{I}_{j k}(t-r)+\frac{2}{r} n_{j} n_{k} \dot{\mathcal{I}}_{j k}(t-r)+\ldots, \\
\hat{\partial}_{j k} B_{j k}\left(\tau_{0}, \boldsymbol{\xi}\right) & =-2 \hat{\partial}_{j k} \ln d \mathcal{I}_{j k}\left(t_{0}-r_{0}\right)+\frac{2}{r_{0}} n_{j} n_{k} \dot{\mathcal{I}}_{j k}\left(t_{0}-r_{0}\right)+\ldots, \\
\hat{\partial}_{i j k} B_{j k}(\tau, \boldsymbol{\xi}) & =-2\left[\mathcal{I}_{j k}(t-r)+\frac{d^{2}}{2 r} \dot{\mathcal{I}}_{j k}(t-r)\right] \hat{\partial}_{i j k} \ln d+\ldots, \\
\hat{\partial}_{i j k} B_{j k}\left(\tau_{0}, \boldsymbol{\xi}\right) & =-2\left[\mathcal{I}_{j k}\left(t_{0}-r_{0}\right)+\frac{d^{2}}{2 r_{0}} \dot{\mathcal{I}}_{j k}\left(t_{0}-r_{0}\right)\right] \hat{\partial}_{i j k} \ln d+\ldots
\end{aligned}
$$


and

$$
\begin{gathered}
\hat{\partial}_{i j k} D_{p q}(\tau, \boldsymbol{\xi})=-2 r \mathcal{I}_{p q}(t-r) \hat{\partial}_{i j k} \ln d-\frac{4 n^{i} n^{j} n^{k}}{d} \dot{\mathcal{I}}_{p q}(t-r)+\ldots \\
\hat{\partial}_{i j k} D_{p q}\left(\tau_{0}, \boldsymbol{\xi}\right)=-2 r_{0} \mathcal{I}_{p q}\left(t_{0}-r_{0}\right) \hat{\partial}_{i j k} \ln d-\frac{4 n^{i} n^{j} n^{k}}{d} \dot{\mathcal{I}}_{p q}\left(t_{0}-r_{0}\right)+\ldots
\end{gathered}
$$

In addition we have

$$
\begin{gathered}
\delta_{Q}(\tau, \boldsymbol{\xi})=\frac{1}{r} k^{p} k^{q} \dot{\mathcal{I}}_{p q}(t-r)+\ldots, \\
\delta_{Q}\left(\tau_{0}, \boldsymbol{\xi}\right)=\frac{1}{r_{0}} k^{p} k^{q} \dot{\mathcal{I}}_{p q}\left(t_{0}-r_{0}\right)+\ldots .
\end{gathered}
$$

We note that the leading terms of the expansions now have the same dependence on the distance of the point of emission of light and of the point of observation from the source of gravitational waves. If the source of light is closer to the source of gravitational waves than the observer, it makes the largest contribution to the effects of time delay and deflection of light.

The asymptotic expansions of integrals (38) - (39) describing propagation of light rays in the static part of the gravitational field of the deflector are:

$$
\begin{gathered}
A(\tau, \boldsymbol{\xi})=-2 \ln d+\ln (2 r)-\frac{d^{2}}{4 r^{2}}+\ldots \\
A\left(\tau_{0}, \boldsymbol{\xi}\right)=-2 \ln d+\ln \left(2 r_{0}\right)-\frac{d^{2}}{4 r_{0}^{2}}+\ldots, \\
B(\tau, \boldsymbol{\xi})=-r-2 r \ln d+r \ln (2 r)-\frac{d^{2}}{2 r}\left[\frac{1}{2}-\ln \left(\frac{d^{2}}{2 r}\right)\right] \ldots \\
B\left(\tau_{0}, \boldsymbol{\xi}\right)=-r_{0}-2 r_{0} \ln d+r_{0} \ln \left(2 r_{0}\right)-\frac{d^{2}}{2 r_{0}}\left[\frac{1}{2}-\ln \left(\frac{d^{2}}{2 r_{0}}\right)\right]+\ldots .
\end{gathered}
$$

These expansions are used for calculation of asymptotic expressions for time delay and the angle of deflection of light rays.

\section{Asymptotic expressions for time delay and the angle of light deflection}

The static part of time delay and deflection angle are given by:

$$
\begin{gathered}
\Delta_{M}=2 \mathcal{M}\left[\ln \left(\frac{r}{r_{0}}\right)+\frac{d^{2}}{4}\left(\frac{1}{r_{0}^{2}}-\frac{1}{r^{2}}\right)\right]+\ldots, \\
\Delta_{S}=\epsilon_{i j p} k^{j} \mathcal{S}^{p} \xi^{i}\left(\frac{1}{r_{0}^{2}}-\frac{1}{r^{2}}\right)+\ldots
\end{gathered}
$$


Expressions for $\alpha_{M}^{i}, \alpha_{S}^{i}$, and $\alpha_{Q}^{i}$ will be the same as in equations (155), (156), and (160) because they are taken at the point of observation only. Expressions for $\beta_{M}^{i}, \beta_{S}^{i}$, and $\beta_{Q}^{i}$ are given at the point of observation by equations (157), (158), and (161). Expressions for $\beta_{M}^{i}, \beta_{S}^{i}$, and $\beta_{Q}^{i}$ at the point of emission of light are given by the same equations (157), (158), and (161) after substituting $r_{0}$ for $r$. The relativistic perturbation $\gamma^{i}$ is calculated in equation (118).

The asymptotic expression for the time delay caused by the quadrupole moment is:

$$
\begin{aligned}
\Delta_{Q}= & -2\left[\mathcal{I}_{i j}(t-r)-\mathcal{I}_{i j}\left(t_{0}-r_{0}\right)\right] \hat{\partial}_{i j} \ln d \\
& +\left(2 n_{i} n_{j}+k_{i} k_{j}\right)\left[\frac{\dot{\mathcal{I}}_{i j}(t-r)}{r}-\frac{\dot{\mathcal{I}}_{i j}\left(t_{0}-r_{0}\right)}{r_{0}}\right]+\ldots .
\end{aligned}
$$

One might think that the effect of retardation is again inversely proportional to the square of impact parameter $d$. However, this is actually true only for sources of gravitational waves with rapidly varying quadrupole moment. If motion of matter inside the localized source of gravitational waves is slow, then conditions (136) apply. In this case, the real amplitude of the effect becomes extremely small, being inversely proportional to $1 / r^{2}$ and $1 / r_{0}^{2}$.

The asymptotic expression for the observed direction $s^{i}$ to the source of light is derived from the basic formula (117) and is:

$$
\begin{aligned}
s^{i}= & K^{i}-\frac{2 r_{0}}{R}\left[\mathcal{I}_{j k}(t-r)-\mathcal{I}_{j k}\left(t_{0}-r_{0}\right)\right] \hat{\partial}_{i j k} \ln d \\
& -\frac{4 k^{j}}{R}\left[\mathcal{I}_{j k}(t-r)-\mathcal{I}_{j k}\left(t_{0}-r_{0}\right)\right] \hat{\partial}_{i k} \ln d \\
& -\frac{2 \xi^{i}}{R}\left[\dot{\mathcal{I}}_{j k}(t-r)-\dot{\mathcal{I}}_{j k}\left(t_{0}-r_{0}\right)\right] \hat{\partial}_{j k} \ln d+\gamma^{i}(\tau, \boldsymbol{\xi})+\ldots,
\end{aligned}
$$

where we have accounted for the approximate equality $R \simeq r-r_{0}$ valid in the case of $\tau_{0}>0$. One can see that deflection angle is small in the expression given. Moreover, if we again assume that motion of the matter is slow, then the observed deflection is even smaller and is inversely proportional to $1 /(r R)$ and $1 /\left(r_{0} R\right)$.

\section{Case 3. Large Impact Parameter}

\section{Asymptotic expansions of independent variables}

In this limiting case we assume that the distance $R$ between observer and source of light is much smaller than $r$ and $r_{0}$, their respective distances from the deflector (see Figure 4 ). Then we have

$$
r_{0}^{2}=r^{2}-2 r R \cos \theta+R^{2}=r^{2}\left(1-\frac{2 R}{r} \cos \theta+\frac{r^{2}}{r^{2}}\right)
$$

which leads to the expansions

$$
\begin{aligned}
& r_{0}=r-R \cos \theta+\ldots, \\
& \frac{1}{r_{0}}=\frac{1}{r}\left(1+\frac{R}{r} \cos \theta\right)+\ldots
\end{aligned}
$$

The time parameters are

$$
\tau=r \cos \theta, \quad \text { and } \quad \tau_{0}=\tau-R .
$$

Their numerical values may be larger or less than zero. The following exact equalities hold: 


$$
\begin{aligned}
d & =r \sin \theta, \\
y & =\tau-r=r(\cos \theta-1), \\
(y r)^{-1} & =\frac{1}{r^{2}(\cos \theta-1)} .
\end{aligned}
$$

In addition, we have asymptotic expansions

$$
\begin{aligned}
y_{0} & =\tau_{0}-r_{0}=y\left(1+\frac{R}{r}\right)+\ldots, \\
\left(y_{0} r_{0}\right)^{-1} & =\frac{1}{y r}+\frac{R}{r^{3}}+\ldots \\
t_{0}-r_{0} & =t-r+R(\cos \theta-1)+\ldots .
\end{aligned}
$$

Thus, we can decompose any function of the time argument $t_{0}-r_{0}$ in a Taylor series with respect to the retarded time $t-r$ if convergence is assumed [62]. For example,

$$
\mathcal{I}_{i j}\left(t_{0}-r_{0}\right)=\mathcal{I}_{i j}(t-r)+R(\cos \theta-1) \dot{\mathcal{I}}_{i j}(t-r)+\ldots .
$$

Finally, we note that the vector $\xi^{i}$ corresponding to impact parameter $d$ can be represented as

$$
\xi^{i}=r\left(N^{i}-k^{i} \cos \theta\right),
$$

where $N^{i}=-K_{0}^{i}=x^{i} / r,|\mathbf{N}|=1$, and $k^{i}$ is the unit vector in the direction from the source of light to observer [63].

\section{Asymptotic expressions for time delay and the angle of light deflection}

In this section all asymptotic expressions for relativistic effects are given only up to leading terms of order $1 / r$ and $1 / r_{0}$. For this reason all residual terms of order $1 / r^{2}$ and $1 / r_{0}^{2}$ are omitted in subsequent formulae without note. Using asymptotic expansions of functions from the previous section and reducing similar terms we obtain

$$
\Delta_{Q}=\frac{1}{1-\cos \theta}\left[k^{i} k^{j}-2 k^{i} N^{j} \cos \theta+\frac{1}{2}\left(1+\cos ^{2} \theta\right) N^{i} N^{j}\right]\left\{\frac{\dot{\mathcal{I}}_{i j}(t-r)}{r}-\frac{\dot{\mathcal{I}}_{i j}\left(t_{0}-r_{0}\right)}{r_{0}}\right\},
$$

where $\cos \theta=\mathbf{k} \cdot \mathbf{N}=\mathbf{K} \cdot \mathbf{K}_{0}$ (see Figures 11 and 4 ). We note that the expression for time delay given above can be further simplified if the definition of "transverse-traceless" tensor with respect to the direction $N^{i}$ is applied [24], [33]:

$$
\mathcal{I}_{i j}^{T T}=\mathcal{I}_{i j}+\frac{1}{2}\left(\delta_{i j}+N_{i} N_{j}\right) N_{p} N_{q} \mathcal{I}_{p q}-\left(\delta_{i p} N_{j} N_{q}+\delta_{j p} N_{i} N_{q}\right) \mathcal{I}_{p q}
$$

where the projection is onto the plane orthogonal to unit vector $N^{i}$. Formula (209) for time delay now assumes the form

$$
\Delta_{Q}=\frac{k^{i} k^{j}}{1-\cos \theta}\left[\frac{\dot{\mathcal{I}}_{i j}^{T T}(t-r)}{r}-\frac{\dot{\mathcal{I}}_{i j}^{T T}\left(t_{0}-r_{0}\right)}{r_{0}}\right] .
$$

Differentiation of $\Delta_{Q}$ with respect to time gives the frequency shift due to a remote localized source of gravitational waves

$$
z_{g}\left(t, t_{0}\right)=1-\frac{d t}{d t_{0}}=-\frac{1}{2} \frac{k^{i} k^{j}}{1-\mathbf{k} \cdot \mathbf{N}}\left[h_{i j}^{T T}(t-r)-h_{i j}^{T T}\left(t_{0}-r_{0}\right)\right],
$$

where the metric $h_{i j}^{T T}$ is defined by the equation $(\mathrm{B} 10)$ and taken in the leading order approximation with respect to $1 / r$. We recognize that the expression (212) is a generalization of the analogous formula for $z_{g}$ obtained previously by Mashhoon \& Seitz [64] in the case of a plane gravitational wave. This exact coincidence demonstrates the power of our formalism, which both reproduces well-known results and yields new observational predictions for relativistic effects in the propagation of light rays in the field of an arbitrary source of gravitational waves 65]. 
Repeating the calculations for the angle of light deflection under the assumption that the wavelength, $\lambda$, of gravitational waves emitted by the localized source is smaller than the distance $R$ between source of light and observer, we come to the following result:

$$
\begin{aligned}
\alpha_{Q}^{i}= & \frac{1}{1-\cos \theta}\left[(\cos \theta-2)\left(k^{i} k^{p} k^{q}+2 k^{i} k^{p} N^{q} \cos \theta\right)+\left(\cos ^{2} \theta-2 \cos \theta-1\right) \times\right. \\
& \left.\times\left(\frac{1}{2} k^{i} N^{p} N^{q} \cos \theta-N^{i} N^{p} N^{q}\right)+N^{i} k^{p} k^{q}-2 N^{i} N^{p} k^{q}\right]\left\{\frac{\ddot{\mathcal{I}}_{p q}(t-r)}{r}\right\} \\
& +2\left(k^{p}-N^{p} \cos \theta\right)\left\{\frac{\ddot{\mathcal{I}}_{i p}(t-r)}{r}\right\} .
\end{aligned}
$$

Transformation of this result using relationship (210) and expression (B10) for $h_{i j}^{T T}$, where only leading terms of order $1 / r$ are retained, reveals that

$$
\alpha_{Q}^{i}=\frac{1}{2} \frac{k^{p} k^{q}}{1-\mathbf{k} \cdot \mathbf{N}}\left[(\mathbf{k} \cdot \mathbf{N}-2) k^{i}+N^{i}\right] h_{p q}^{T T}(t-r)+k^{p} h_{i p}^{T T}(t-r)
$$

and, because the vector $\beta^{i}$ is small,

$$
s^{i}=K^{i}+\alpha_{Q}^{i}+\gamma^{i}+\ldots
$$

where the ellipsis designates unimportant terms of higher order with respect to $1 / r$ [66], and we have neglected the constant deflection caused by mass-monopole and spin-dipole dependent terms. One sees again that only the transverse-traceless component $h_{i j}^{T T}$ of the metric tensor appears in the final expression.

It is worthwhile to stress that the observed optical direction to the source of light given by the formula (215) coincides with that which can be obtained by means of VLBI observations. Indeed, it is easy to confirm that equation (215) can be re-written as follows 67]

$$
s^{i}=K^{i}+\frac{1}{2} \frac{K^{i}+N^{i}}{1+\mathbf{K} \cdot \mathbf{N}} K^{p} K^{q} h_{p q}^{T T}(t-r)-\frac{1}{2} K^{p} h_{i p}^{T T}(t-r) .
$$

The direction to the source of electromagnetic waves measured by VLBI is determined as difference between times of arrival of the wave to the first and second antennas. Taking into account equations (94) and (211) for the first and second observing sites, and assuming that the time difference $t_{2}-t_{1}$ in observation of the radio signal at the observatories is small compared to the period of gravitational waves, we find

$$
t_{2}-t_{1}=-\left(\mathbf{K}+\frac{1}{2} \frac{K^{i}+N^{i}}{1+\mathbf{K} \cdot \mathbf{N}} K^{p} K^{q} h_{p q}^{T T}(t-r)\right) \cdot\left(\mathbf{x}_{2}-\mathbf{x}_{1}\right) .
$$

If the baseline vector measured in the local inertial frame is denoted as $\mathbf{b}$ and the transformation (106) is taken into account,

$$
x_{2}^{i}-x_{1}^{i}=b^{i}-\frac{1}{2} h_{i j}^{T T}(t-r) b^{j}+O\left(\mathbf{b}^{2}\right)
$$

We confirm that

$$
t_{2}-t_{1}=-\mathbf{s} \cdot \mathbf{b}
$$

where the vector $s^{i}$ is given by formula (216), which proves our statement. It is worth emphasizing that equation (216) was obtained independently by Pyne et al. ( $\sqrt{16}$, see formula (47)). Their formalism, however, has a limited region of application. Extension of the formalism of Pyne et al. [16] was one of the motivation of the present work. 


\section{CONCLUSIONS}

The most accurate astrometric measurements are differential. They measure the angle between 2 sources. The highest accuracy is attainable when the sources are close to each other in the sky. In contrast, angular deflection by gravitational waves varies only over large angles in the general case of large impact parameter. Specifically, in such a case the bending angle depends only on the metric in the neighborhood of the observer and its first derivatives, as in equations (214), (215). It thus can vary only as a quadrupole and the derivative of a quadrupole, over the sky. Similarly, equations (123), (160) and (195) depend on the mass quadrupole moment $\mathcal{I}_{i j}$ and its first and second derivatives. Note that the the angle of light deflection (123) involves the time integrals of $\mathcal{I}_{i j}(t-r)$ which may be interpreted as the presence of the "kinematic resonance effect" [14]; however, this term is small, as discussed above. In the context of a purely locally-determined deflection angle, it is not unexpected that lines of sight that pass close to the deflector show almost purely the static effect, as was shown in section 7 .

The magnitudes of the leading terms in the limiting forms for the deflection angle $\alpha_{Q}$, in equations (123), (160) and (195) are $\alpha_{Q} \sim \Omega^{2} G M a^{2} / c^{4} r$, where $M$ is the mass of the deflector, and $a$ is its dimension. The frequency of the gravitational waves is $\Omega$. For a gravitationally bound binary system with a circular orbit, $\Omega$ is twice the orbital frequency 68]. We can use Kepler's third law to express this in the form $\alpha_{Q} \sim \Omega^{2 / 3}(G M)^{5 / 3} / c^{4} r$, or alternatively, $\alpha_{Q} \sim 2.4 \times 10^{-14}\left(M / M_{\odot}\right)^{5 / 3} P_{\mathrm{sec}}^{-2 / 3}\left(r_{\mathrm{kpc}}\right)^{-1}$ arcsec where $P_{\mathrm{sec}}$ is the orbital period of the binary system. For a contact white-dwarf binary at $200 \mathrm{pc}$, the expected deflection is about $7 \times 10^{-13}$ arcsec, with a period of about 1000 sec. For a supermassive black-hole binary, with mass $10^{6} M_{\odot}$ and period 10 yr at a distance of 1 Mpc, the expected deflection is about $5 \times 10^{-11}$ arcsec.

Because the effect varies smoothly over the sky, the presently available astrometric accuracies are a few microarcseconds. Higher accuracies are attainable only over smaller angles. Very-long baseline interferometry of a suite of radio sources attains microarcsecond accuracy, over periods of days to years. Specially-designed observations sensitive to source motions of minutes or hours might attain higher accuracy, perhaps as much as an order of magnitude better. Clearly, detection of deflection of light rays by gravitational waves from nearby localized sources is not a goal for the near future because of its smallness. However the background gravitational wave noise may be, perhaps, measured.

The near-perfect cancellation of the effect in General Relativity suggests that deflection of light by gravitational waves could be a test of that theory in radiative regime [70]. In a theory that does not posess the symmetries that cause the deflection to vanish, we can only guess the resulting deflection. Such a guess might multiply the generalrelativistic $\alpha_{Q}^{i}$ by 3 factors. The first factor, of $r / d$, reflects the amplitude of the gravitational wave at the point of closest approach, rather than at the observer. The second factor, some function of the distance to the source

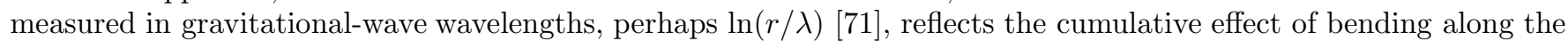
line of sight. The final factor, unknown, reflects the coupling of the non-general-relativistic part of the wave to the source and its effect on the light ray. For a source an arcsecond from the deflectors described above, the first 2 factors can increase the effect by several orders of magnitude. Moreover, if the effect is not local to the observer, differential astrometry across small angles can detect it, so that greater accuracy is attainable. Given sufficiently strong departures from General Relativity, the effect might be detectable.

\section{ACKNOWLEDGMENTS}

We are greatful to V.B. Braginsky, M.V. Sazhin, and P. Schneider for valuable and stimulating discussions. S.M. Kopeikin is pleasured to acknowledge the hospitality of G. Neugebauer and G. Schäfer and other members of the Institute for Theoretical Physics of the Friedrich Schiller University of Jena.

The US National Science Foundation supported parts of this work (AST-9731584). This work was partially supported by the Thüringer Ministerium für Wissenschaft, Forschung und Kultur grant No B501-96060 and by the Max-Planck-Gesellschaft grant No 02160-361-TG74.

\section{APPENDIX A: COMPARISON TO THE PAPER BY DAMOUR \& ESPOSITO-FARĖSE}

In this Appendix we rederive the results of the paper by Damour \& Esposito-Farèse 23 applying the generalized isotropic ADM coordinate conditions (For an application of the conditions in post-Newtonian calculations, see e.g. [41]). This explicitly shows that the asymptotic results do not depend on the chosen gauge. We do not put $c=1$ in this and the following appendices to make more clear the order of terms with respect to the small parameter $1 / c$.

The ADM coordinate conditions, in linear approximation, read 


$$
2 \nabla_{i} g_{0 i}-\nabla_{0} g_{i i}=0, \quad 3 \nabla_{j} g_{i j}-\nabla_{i} g_{j j}=0
$$

where $\nabla_{0}=\partial / \partial t$ and $\nabla_{i}=\partial / \partial x^{i}$. For comparison, the harmonic coordinate conditions, in linear approximation, read:

$$
2 \nabla_{i} g_{0 i}-\nabla_{0} g_{i i}=\nabla_{0} g_{00}, \quad 2 \nabla_{j} g_{i j}-\nabla_{i} g_{j j}=-\nabla_{i} g_{00}
$$

The ADM gauge conditions (A1) brings the space-space component of metric to the form

$$
g_{i j}=\delta_{i j}\left(1+\frac{1}{3} h_{k k}\right)+h_{i j}^{T T}
$$

where $h_{i j}^{T T}$ denotes the transverse-traceless part of $h_{i j}$. Furthermore, in linear approximation, the Einstein field equations read

$$
\begin{aligned}
h_{00} & =-\frac{8 \pi}{c^{4}} \Delta^{-1}\left(T_{00}+T_{i i}\right), \\
h_{0 i} & =-\frac{16 \pi}{c^{4}} \Delta^{-1}\left(T_{0 i}-\frac{1}{4} \nabla_{0} \nabla_{i} \Delta^{-1} T_{00}\right), \\
h_{k k} & =-\frac{24 \pi}{c^{4}} \Delta^{-1} T_{00}, \\
h_{i j}^{T T} & =-\frac{16 \pi}{c^{4}} P_{i j k l} \square_{r e t}^{-1} T_{k l},
\end{aligned}
$$

where the TT-projection operator $P_{i j k l}$, applied to symmetric tensors depending on both time and spatial coordinates, is given by

$$
P_{i j k l}=\left(\delta_{i k}-\Delta^{-1} \nabla_{i} \nabla_{k}\right)\left(\delta_{j l}-\Delta^{-1} \nabla_{j} \nabla_{l}\right)-\frac{1}{2}\left(\delta_{i j}-\Delta^{-1} \nabla_{i} \nabla_{j}\right)\left(\delta_{k l}-\Delta^{-1} \nabla_{k} \nabla_{l}\right)
$$

where $\Delta^{-1}$ denotes the Euclidean inverse Laplacian.

We now follow the calculation in the paper by Damour \& Esposito-Farèse 23] using the ADM coordinate conditions. The deflection of the light ray is given by

$$
\Delta l_{\mu}=l_{\mu}(+\infty)-l_{\mu}(-\infty)=\frac{1}{2} \int_{-\infty}^{+\infty} d \tau l^{\alpha} l^{\beta} \nabla_{\mu} h_{\alpha \beta}\left(\xi^{\lambda}+\tau l^{\lambda}\right) .
$$

In terms of the Fourier transform

$$
\hat{h}_{\mu \nu}\left(k^{\lambda}\right)=\int d^{4} x h_{\mu \nu}\left(x^{\lambda}\right) \mathrm{e}^{-i k_{\alpha} x^{\alpha}}
$$

where $k_{\alpha} x^{\alpha}=-k^{0} x^{0}+\mathbf{k} \cdot \mathbf{x}$, the boldface letters denote spatial components of vectors [72], and integration is over all of space-time. Accounting for the formulae (A10) the equation for light deflection now reads

$$
\Delta l_{\mu}=i \pi \int \frac{d^{4} k}{(2 \pi)^{4}} k_{\mu} \mathrm{e}^{i k_{\mu} \xi^{\mu}} l^{\alpha} l^{\beta} \hat{h}_{\alpha \beta}\left(k^{\lambda}\right) \delta\left(\mathbf{k} \cdot \mathbf{l}-k^{0} l^{0}\right),
$$

where use has been made of the exact relationship

$$
\int_{-\infty}^{+\infty} d \tau \exp \left(i \tau k_{\alpha} l^{\alpha}\right)=2 \pi \delta\left(\mathbf{k} \cdot \mathbf{l}-k^{0} l^{0}\right)
$$

In terms of the Fourier transformed energy-momentum tensor $\hat{T}_{\alpha \beta}$, the Fourier transformed metric field reads 


$$
\begin{aligned}
& \hat{h}_{00}=\frac{8 \pi}{c^{4}} \frac{\hat{T}_{00}+\hat{T}_{i i}}{\mathbf{k}^{2}} \\
& \hat{h}_{0 i}=\frac{16 \pi}{c^{4}}\left(\frac{\hat{T}_{0 i}}{\mathbf{k}^{2}}-\frac{1}{4} \frac{k_{0} k_{i}}{\mathbf{k}^{2}} \frac{\hat{T}_{00}}{\mathbf{k}^{2}}\right) \\
& \hat{h}_{k k}=\frac{24 \pi}{c^{4}} \frac{\hat{T}_{00}}{\mathbf{k}^{2}} \\
& \hat{h}_{i j}^{T T}=\frac{16 \pi}{c^{4}}\left[\left(\delta_{i l}-\frac{k_{i} k_{l}}{\mathbf{k}^{2}}\right)\left(\delta_{j k}-\frac{k_{j} k_{k}}{\mathbf{k}^{2}}\right)-\frac{1}{2}\left(\delta_{i j}-\frac{k_{i} k_{j}}{\mathbf{k}^{2}}\right)\left(\delta_{k l}-\frac{k_{k} k_{l}}{\mathbf{k}^{2}}\right)\right] \frac{\hat{T}_{k l}}{\mathbf{k}^{2}-\left(k^{0}+i \epsilon\right)^{2}},
\end{aligned}
$$

where $\epsilon$ is a positive infinitesimal number which shows explicitly that we have used the retarded Green's function while solving the Einstein equations for the component of the metric $h_{i j}$. If we put $\mathbf{k} \cdot \mathbf{l}-k^{0} l^{0}=0$ and take into account the relations

$$
\hat{T}_{00}=\frac{k_{i} k_{j}}{k_{0}^{2}} \hat{T}_{i j}, \quad \hat{T}_{0 i}=\frac{k_{j}}{k_{0}} \hat{T}_{i j}
$$

which follow from the macroscopic equations of motion for matter $\nabla_{\nu} T^{\mu \nu}=0$, we find:

$$
l^{\alpha} l^{\beta} \hat{h}_{\alpha \beta}=\left(l^{0}\right)^{2}\left(\frac{k_{i}}{k^{0}}-\frac{l_{i}}{l^{0}}\right)\left(\frac{k_{j}}{k^{0}}-\frac{l_{j}}{l^{0}}\right) \frac{\hat{T}_{i j}}{\mathbf{k}^{2}-\left(k^{0}+i \epsilon\right)^{2}} .
$$

This expression is identical with that obtained by Damour \& Esposito-Farèse [23] in the harmonic gauge. For this reason calculation of the total deflection angle gives the same result in both harmonic and ADM gauges, reflecting the coordinate independence of the final result.

Defining $\alpha_{\mu}=\Delta l_{\mu} / l^{0}$, one gets for the angle of total deflection

$$
\begin{aligned}
& \alpha_{1}=-\frac{4}{d^{3}}\left[\mathcal{I}_{11}\left(t^{*}\right)-\mathcal{I}_{22}\left(t^{*}\right)\right], \\
& \alpha_{2}=\frac{8}{d^{3}} \mathcal{I}_{12}\left(t^{*}\right), \\
& \alpha_{3}=\alpha^{0}=-\frac{2}{d^{2}}\left[\dot{\mathcal{I}}_{11}\left(t^{*}\right)-\dot{\mathcal{I}}_{22}\left(t^{*}\right)\right] .
\end{aligned}
$$

One can see that expressions for $\alpha_{1}, \alpha_{2}$ are the same as those obtained in section 7.1.2. The quantity $\alpha^{0}$ gives the gravitational shift in frequency of the electromagnetic wave. It can be obtained from the expression for gravitational time delay $\Delta_{Q}$ after its differentiation with respect to time.

\section{APPENDIX B: HARMONIC AND ADM GAUGE CONDITIONS IN THE FIRST POST-MINKOWSKIAN APPROXIMATION}

In this Appendix we give other representations of the metric coefficients (12) - (14). Using the ADM coordinate conditions (A1) of Appendix A the metric coefficients (12) - (14) can be cast into the "canonical" ADM form

$$
\begin{aligned}
& h_{00}^{a d m}=\frac{2 \mathcal{M}}{c^{2} r}+\frac{\mathcal{I}_{i j}(t)}{c^{2}} \nabla_{i} \nabla_{j} r^{-1}, \\
& h_{0 i}^{a d m}=-\frac{2}{c^{3}} \frac{\epsilon_{i p q} \mathcal{S}_{p} N_{q}}{r^{2}}+\frac{2 \dot{\mathcal{I}}_{i j}(t)}{c^{3}} \nabla_{j} r^{-1}-\frac{\dot{\mathcal{I}}_{j k}(t)}{4 c^{3}} \nabla_{i} \nabla_{j} \nabla_{k} r, \\
& h_{k k}^{a d m}=3 h_{00},
\end{aligned}
$$




$$
\begin{aligned}
h_{i j}^{a d m T T}= & \frac{2 \ddot{\mathcal{I}}_{i j}(t-r / c)}{c^{4} r} \\
& +\left(\delta_{i j} \nabla_{k} \nabla_{l}-2 \delta_{i l} \nabla_{j} \nabla_{k}-2 \delta_{j l} \nabla_{i} \nabla_{k}\right)\left[\frac{\mathcal{I}_{k l}(t-r / c)}{c^{2} r}-\frac{\mathcal{I}_{k l}(t)}{c^{2} r}\right]+ \\
& \nabla_{i} \nabla_{j} \nabla_{k} \nabla_{l}\left[\frac{{ }^{(-2)} \mathcal{I}_{k l}(t-r / c)}{r}-\frac{{ }^{(-2)} \mathcal{I}_{k l}(t)}{r}-\frac{\mathcal{I}_{k l}(t) r}{2 c^{2}}\right],
\end{aligned}
$$

where we have used a special symbolic notation for "semihereditary functionals" 73

$$
{ }^{(-1)} \mathcal{I}_{i j}(t) \equiv \int_{-\infty}^{t} d v \mathcal{I}_{i j}(v), \quad{ }^{(-2)} \mathcal{I}_{i j}(t) \equiv \int_{-\infty}^{t} d v^{(-1)} \mathcal{I}_{i j}(v)
$$

The following equality holds: ${ }^{(-2)} \ddot{\mathcal{I}}_{i j}(t-r)=\mathcal{I}_{i j}(t-r)$. We also notice that $\Delta\left(\mathcal{I}_{i j}(t-r / c) / r\right)=\ddot{\mathcal{I}}_{i j}(t-r / c) / c^{2} r$ for $r \neq 0$. For this reason function ${ }^{(-2)} \ddot{\mathcal{I}}_{i j}(t-r)$ is a solution of the homogeneous d'Alembert's equation; that is, $\square\left[{ }^{(-2)} \mathcal{I}_{i j}(t-r) / r\right]=0$ for $r \neq 0$.

We emphasize that the metric (B1)-(B4) is an external solution of the equations (A4)-(A7), outside the source of gravitational waves. It matches smoothly to the internal solution, which is valid inside the source, without additional coordinate transformations. It is remarkable that outside the source the metric component (B4) may be represented as an algebraic decomposition of the retarded and instantaneous functions of time

$$
h_{i j}^{a d m T T}=h_{i j}^{T T}\left(t-\frac{r}{c}, \mathbf{x}\right)+\tilde{h}_{i j}(t, \mathbf{x}),
$$

where $h_{i j}^{T T}(t-r / c, \mathbf{x})$ is shown below in $(\mathrm{B} 10)$, and $\tilde{h}_{i j}(t, \mathbf{x})$ is the rest of the metric component $h_{i j}^{a d m T T}$ which is actually a symmetrized gradient of a vector comprising of singular harmonic functions. For this reason function $\tilde{h}_{i j}(t, \mathbf{x})$ satisfies the condition $P_{i j k l} \tilde{h}_{k l}(t, \mathbf{x}) \equiv 0$ and can be eliminated by an infinitesimal coordinate transformation. Making use of this and without leaving the ADM coordinate conditions, we may construct the following representation for the metric:

$$
\begin{aligned}
h_{00}= & \frac{2 \mathcal{M}}{c^{2} r} \\
h_{0 i}= & -\frac{2}{c^{3}} \frac{\epsilon_{i p q} \mathcal{S}_{p} N_{q}}{r^{2}}, \\
h_{k k}= & 3 h_{00}, \\
h_{i j}^{T T}= & \frac{2 \ddot{\mathcal{I}}_{i j}(t-r / c)}{c^{4} r}+ \\
& \left(\delta_{i j} \nabla_{k} \nabla_{l}-2 \delta_{i l} \nabla_{j} \nabla_{k}-2 \delta_{j l} \nabla_{i} \nabla_{k}\right) \frac{\mathcal{I}_{k l}(t-r / c)}{c^{2} r}+\nabla_{i} \nabla_{j} \nabla_{k} \nabla_{l}\left[\frac{{ }^{(-2)} \mathcal{I}_{k l}(t-r / c)}{r}\right] .
\end{aligned}
$$

This form of the metric is obtained from the expressions (B1)- $B 4$ by applying the coordinate transformation [74]

$$
\begin{aligned}
& w^{0}=\frac{1}{2} \nabla_{k} \nabla_{l}\left[\frac{{ }^{(-1)} \mathcal{I}_{k l}(t)}{c r}\right], \\
& w^{i}=\frac{1}{2} \nabla_{i} \nabla_{k} \nabla_{l}\left[\frac{{ }^{(-2)} \mathcal{I}_{k l}(t)}{r}\right]-2 \nabla_{k}\left[\frac{\mathcal{I}_{k i}(t)}{c^{2} r}\right]+\frac{1}{4} \nabla_{i} \nabla_{k} \nabla_{l}\left[\frac{\mathcal{I}_{k l}(t) r}{c^{2}}\right] .
\end{aligned}
$$

It is marvelous that this representation of metric also fulfils the harmonic coordinate conditions (A2). This means that outside the localized source of gravitational waves the class of ADM coordinates overlaps with that of harmonic ones. The coordinate transformation from metric (12)-(14) written in the pure harmonic coordinate system to the ADM-harmonic metric (B7)-(B10) is:

$$
\begin{aligned}
& w^{0}=\frac{1}{2} \nabla_{k} \nabla_{l}\left[\frac{{ }^{(-1)} \mathcal{I}_{k l}(t-r / c)}{c r}\right], \\
& w^{i}=\frac{1}{2} \nabla_{i} \nabla_{k} \nabla_{l}\left[\frac{{ }^{(-2)} \mathcal{I}_{k l}(t-r / c)}{r}\right]-2 \nabla_{k}\left[\frac{\mathcal{I}_{k i}(t-r / c)}{c^{2} r}\right] .
\end{aligned}
$$


These gauge functions have been extensively used in the main body of the paper for elaborating unique interpretation of observable effects. In contrast to the expressions (B1)-(B4) the expressions (B6)-(B9) show terms which decay like $1 / r^{4}$ and $1 / r^{5}$. These terms depend on time integrals of the quadrupole moment $\mathcal{I}_{i j}(t-r / c)$ and may lead to the appearance of the "kinematic resonance effect" discussed by Braginsky \& Grishchuk [14]. Another important remark is that the transformations (B13)-(B14) clearly show how to eliminate non-radiative terms from the metric, written down in a harmonic gauge, including all terms with respect to any power of $1 / r$. Previously used transformations (see, for example, the textbook of Misner et al. [45], paragraph 35) dealt only with terms of the first order in $1 / r$ and could not be applied for analysis of gravitational radiation in near or intermediate zones of the localized source of gravitational waves.

[1] Taylor, J.H. 1993, Class. Quantum Grav., 10, 167

[2] Thorne, K.S. 1995, "Gravitational Waves", In: Proc. of the Snowmass 95 Summer Study on Particle and Nuclear Astrophysics and Cosmology, eds. E. W. Kolb \& R. Peccei, World Scientific: Singapore, p. 398

[3] Schutz, B. 1997, In: Proc. of the Alpbach Summer School 1997 "Fundamental Physics in Space", ed. A. Wilson, ESA Publications Division, Noordwijk: The Netherlands, SP-420, p. 229

[4] Warner, B. 1995, Ap\&SS, 2525, 249

[5] Hils, D., Bender, P., \& Webbink, R.F. 1990, Astrophys. J. , 360, 75

[6] Rajagopal, M., \& Romani, R.W. 1995, Astrophys. J. , 446, 543

[7] Sazhin, M.V. 1978, Sov. Astron., 22(1), 36

[8] Sazhin, M.V., \& Saphonova, M. V. 1993, Astron. Astrophys. Space Sci., 208, 93

[9] Wahlquist, H. 1987, Gen. Rel. Grav., 19, 1101

[10] Estabrook, F. B., \& Wahlquist, H. D., 1975, GRG, 6, 439

[11] Tinto, M. 1996, Phys. Rev. D , 53, 5354

[12] Bertotti, B., Vecchio, A. \& Iess L., 1998, preprint gr-qc/9806021

[13] Braginsky, V. B., Kardashev, N. S., Polnarev, A. G., \& Novikov, I. D. 1990, Nuovo Cim. B, 105,1141

[14] Braginsky, V. B., \& Grishchuk, L. P., 1985, Soviet Physics - JETP, 62, 427

[15] Kaiser, N., \& Jaffe, A. 1997, Astrophys. J. , 484, 545

[16] Pyne, T., Gwinn, C.R., Birkinshaw, M., Eubanks, T.M., \& Matsakis, D.N. 1996, Astrophys. J. , 465,566

[17] Gwinn, C.R., Eubanks, T.M., Pyne, T., Birkinshaw, M. \& Matsakis, D.N. 1997, Astrophys. J. , 485, 87

[18] Montanari, E., 1998, Class. Quant. Grav., 15, 2493

[19] Fakir, R. 1994, Astrophys. J. , 426, 74; Phys. Rev. D , 50, 3795

[20] Durrer, R. 1994, Phys. Rev. Lett. , 72, 3301

[21] Labeyrie, A., 1993, A\&A, 268, 823

[22] Pogrebenko, S., Mingaliev, M., Montebugnoli, S., Neizvestny, S., Borisov, N., \& Stolyarov, V., 1994, GPS QSO 2022+171 - A Possible Target for VLBI Detection of Gravitational Waves, In: Proc. 2-nd EVN/JIVE Symposium, Toruń, p. 33

[23] Damour, T., \& Esposito-Farèse, G. 1998, Phys.Rev. D58, 042001

[24] Kopeikin, S.M. 1997, J. Math. Phys., 38, 2587

[25] Bartel, N., Herring, T.A., Ratner, M.I., Shapiro, I.I., \& Corey, B.E. 1986, Nature, 319, 733

[26] Gwinn, C.R., Moran, J.M., Reid, M.J., \& Schneps, M.H. 1988, Astrophys. J. , 330, 817

[27] Greenhill, L.J., Moran, J.M., Reid, M.J., Menten, K.M., \& Hirabayashi, H. 1993, Astrophys. J. , 406,482

[28] Peterson, D., \& Shao, M. 1997, In: Proc. of the ESA Symposium "Hipparcos, Venice 97," ed. B. Battrick, ESA Publications Division, Noordwijk: The Netherlands, p. 789

[29] Lindegren, L., and Perryman, M.A.C., 1996, A\&AS, 116, 579

[30] Gai, M., Bertinetto, F., Bisi, M., Canuto, E., Carollo, D., Cesare, S., Lattanzi, M. G., Mana, G., Thomas, E., \& Viard, T. 1997, In: Proc. of the ESA Symposium "Hipparcos, Venice 97," ed. B. Battrick, ESA Publications Division, Noordwijk: The Netherlands, p. 835

[31] Brumberg, V.A. 1991, Essential Relativistic Celestial Mechanics, Adam Hilger: Bristol

[32] Klioner, S.A., \& Kopeikin, S.M. 1992, Astrophys. J. , 104, 897

[33] Thorne, K.S. 1980, Rev. Mod. Phys., 52, 299

[34] Blanchet, L., \& Damour, T. 1986, Phil. Trans. R. Soc. London, A320, 379

[35] Blanchet, L., \& Damour, T. 1989, Ann. Inst. Henri Poincaré, 50, 377

[36] Hereafter we put the universal gravitational constant $G=1$. Repeated spatial indices in all subsequent equations mean summation from 1 to 3 .

[37] Arnowitt, R., Deser, S., \& Misner C.W., 1962, The dynamics of General Relativity, In: Gravitation: an introduction to current research, ed. L. Witten, John Wiley \& Sons, Inc.: New York, p. 227 
[38] Fock (1959) proved that the harmonic conditions, together with the requirements of "Euclidean" behavior at infinity and of a unique wave-type solution, determine the coordinate system uniquely apart from a Lorentz transformation. However, the transformations (9), (10) are not of this type which seems to contradict the statement made by Fock. The contradiction is resolved by noticing that Fock always worked with regular functions and implicitly assumed that harmonic coordinates cover the entire space-time. The harmonic metric (6)-(8) is, in contrast to Fock's one, singular at the origin of the coordinate system. It is this property which allows us to tremendously extend the class of harmonic coordinate transformations, not restricting them to Lorentz boosts alone.

[39] Fock, V.A., 1959, The Theory of Space, Time and Gravitation, Pergamon Press: London

[40] Coope, J.A.R., Snider, R.F., \& McCourt, F.R. 1965, J. Chem. Phys., 43, 2269

[41] Schäfer, G. 1985. Ann. Phys. (N.Y.), 161, 81

[42] We draw attention to the minus sign in front of the function $k^{i} w^{0}$ in equation (36). It appears because of the equality $w_{0}=-w^{0}$, for we raise and lower space-time indices using the Minkowski metric $\eta_{\alpha \beta}=\operatorname{diag}(-1,+1,+1,+1)$. Spatial indices are raised and lowered by means of the Kronecker symbol $\delta_{i j}$. For this reason, there is no difference between upper and lower spatial indices.

[43] In fact, we must first calculate the derivative and then integrate with respect to time. This eliminates the divergent part of the integral (38).

[44] Damour \& Esposito-Farèse [23] have used in their calculations of light deflection the space-time Fourier transform of metric tensor. In our approach we use a Fourier transform in the time domain only. This is simpler than the approach of Damour \& Esposito-Farèse 23] and is complete enough for calculations of observable effects.

[45] Misner, C.W., Thorne, K.S., \& Wheeler, J.A., 1973, Gravitation, W. H. Freeman and Company, New York

[46] In higher approximations the outgoing gravitational radiation leads to secular decrease in $\mathcal{M}$ and $\mathcal{S}$. We neglect this effect in the present paper.

[47] Gradshteyn, I.S., \& Ryzhik, I.M., 1994, Tables of Integrals, Series, and Products, Academic Press: San Diego

[48] Note the usage of the "hat" derivatives as in equation (36).

[49] We emphasize once again that vectors $K^{i}$ and $K_{0}^{i}$ defined in section 4.1 determine different directions, as shown in Fig. 1.

[50] We note that, in general, this distance depends on time. The difficulty is that the observer and source of light are freely falling particles with respect to the source of gravitational waves. Hence, their motion should be calculated by solving the equations of motion of the particles under the influence of the gravitational field of the source. This makes the coordinates of the particles dependent on time. However, if distances from observer and source of light to the source of gravitational wave are large enough we can neglect this time dependence. Indeed, in the ADM-harmonic gauge the main contribution to the gravitational force perturbing the particles's motion is proportional to $G M / r^{2}$ and is caused by the $g_{00}$ component of the metric tensor. This static, Newtonian force produces only extremely weak, constant acceleration of the particles. Thus, it is easy to see that the gravitational-wave part of the metric tensor will produce effects of second order with respect to $G$ in the motion of the particles. In what follows we assume that distance $\left|\mathbf{x}-\mathbf{x}_{0}\right|$ is constant.

[51] Landau, L.D. \& Lifshitz, E.M. 1962, The Classical Theory of Fields, Pergamon Press: Oxford

[52] LISA Pre-Phase A Report, Second Edition, 1998, Preprint MPQ 233, also available on ftp server: ftp.ippgarching.mpg.de/pub/grav/lisa/ppa2.05.ps.gz

[53] The case of observer moving with respect to the ADM-harmonic system with velocity $v^{i}$ may be considered after completing the additional Lorentz transformation using the well-known procedure (see, for example, 31]).

[54] Terms proportional to $1 / r_{0}$ do not appear in the expansion (159) while terms of order $1 / r_{0}^{2}$ do appear. The situation is different, however, if the impact parameter is large (see section 7.2.3).

[55] Weinberg, S. 1972, Gravitation and Cosmology, John Wiley \& Sons: New York

[56] Usually, it is assumed that the quadrupolar approximation preassumes a slow-motion approximation [23], 55]. We would like to emphasize, however, that the slow-motion approximation is not a direct consequence of the quadrupolar approximation which only relies on the assumption that the magnitudes of the octupole and other higher multipoles are much smaller than that of the quadrupole. In principle, this does not prohibit velocities of matter to be close to velocity of light.

[57] Those residual terms in this formulae which depend only on the quadrupole moment decay as either $1 / r^{2}$, or $1 / r_{0}^{2}$. It is remarkable that both terms of order $1 / r$ and those of $1 / r_{0}$ do completely vanish because of cancellation of corresponding terms.

[58] Schneider, P., Ehlers, J., \& Falco, E. E. 1992, Gravitational Lensing, Berlin: Springer

[59] Wex, N., \& Kopeikin, S.M., 1998, ApJ, in press

[60] Ibáñez, J., \& Martin, J., 1982, Phys. Rev. D , 26, 384

[61] Ibáñez, J., 1983, A\&A, 124, 175

[62] A necessary condition for convergence is the assumption that the distance $R$ between source of light and observer is smaller than the wavelength $\lambda$ of gravitational waves emitted by the localized source, that is $R \ll \lambda$. This condition holds, for instance, for ultra-long gravitational waves of cosmological origin.

[63] We recall that the origin of the coordinate system is at the center of mass of the localized source emitting gravitational waves. This explains the appearance of the minus sign in formula (208).

[64] Mashhoon, B., \& Seitz, M. 1991, MNRAS, 249, 84

[65] We can even apply our formalism to a general theoretical treatment of the output of ground-based or space-borne grav- 
itational wave detectors such as LIGO or LISA. For instance, if we introduce the notation $\Delta R=c \Delta_{Q}$ for the variation of distance between the test bodies of the detectors, then from formula (211) and Taylor expansion of the quadrupole moment 207) one immediately obtains the well known result

$$
\frac{\Delta R}{R}=\frac{1}{2} k^{i} k^{j} h_{i j}^{T T}(t-r)+O\left(\frac{R}{r}\right)+O\left(\frac{R}{\lambda}\right),
$$

where $\lambda$ is the wavelength of gravitational waves impinging on the detector. If necessary we can write down corrections to this formula up to any order. The case when $\lambda$ is comparable with $R$ should be treated in a slightly different way and is a matter for a separate study. We emphasize that this can be done in the framework of our formalism as well.

[66] These terms are proportional to the first time derivative of the metric perturbations $h_{i j}^{T T}(t-r)$.

[67] Let us draw attention to the fact that formula $(216)$ is applicable to an arbitrary source of gravitational waves, including the case of a plane gravitational wave, as has been debated in the literature (see, for example, [13], [15] and references therein). Unfortunately there has been no agreement among the previous authors regarding explicit expression for the light deflection angle. Formula (216) fill in this gap and closes the debate.

[68] Tsvetkov \& Tsyrulev [69] showed the presence of a frequency $\Omega / 2$ in the spectrum of gravitational radiation emitted by a rapidly rotating fluid ball of a magnetized homogeneous gravitating liquid which has an asymmetric form due to magnetic strains. We do not expect any such sources to be prominent at low frequencies.

[69] Tsvetkov, V. P., \& Tsyrulev, A. N., 1983, Sov. Astron., 27, 66

[70] Damour, T., \& Esposito-Farèse, G. 1992, Class. \& Quant. Grav., 9, 2093

[71] Faraoni, V., \& Gunzig, E., 1998, A\&A, 332, 1154

[72] For example, $\mathbf{k}=k^{i}=\left(k^{1}, k^{2}, k^{3}\right)$.

[73] Blanchet, L., \& Damour, T. 1988, Phys. Rev. D , 37, 1410

[74] We recall that $h_{\mu \nu} \rightarrow h_{\mu \nu}+\nabla_{\nu} w_{\mu}+\nabla_{\mu} w_{\nu}$ and indices are raised and lowered with the help of the Minkowski metric $\eta_{\alpha \beta}=$ $\operatorname{diag}(-1,+1,+1,+1)$. The spatial part of the transformation $\nabla_{\nu} w_{\mu}+\nabla_{\mu} w_{\nu}$ also satisfies the identity $P_{i j k l}\left(\nabla_{k} w_{l}+\nabla_{l} w_{k}\right) \equiv$ 0 as does the function $\tilde{h}_{i j}(t, \mathbf{x})$. For this reason the TT-part of the metric (B4) can be transformed into (B10) by means of the coordinate transformation (B12). This explicitly reveals that the decomposition of the spatial component of the metric tensor into TT-, T-, and L-parts [37] is not unique outside the source of gravitational waves. This non-uniqueness appears because outside the source the metric involves singular (at the origin of coordinate system) harmonic functions proportional to partial derivatives of $F(t) / r$, which are solutions of the homogeneous Laplace equation. Similar remarks apply to the transformation (B14). We note that the approximation of order $G^{3}$ an analogous non-uniqueness of the ADM coordinate conditions is related to a fundamental ambiguity of the dynamics of point-like masses which are treated as singularities in the metric tensor 75 .

[75] Jaranowski, P., \& Schäfer, G., 1998, Phys. Rev. D , 57, 7274 


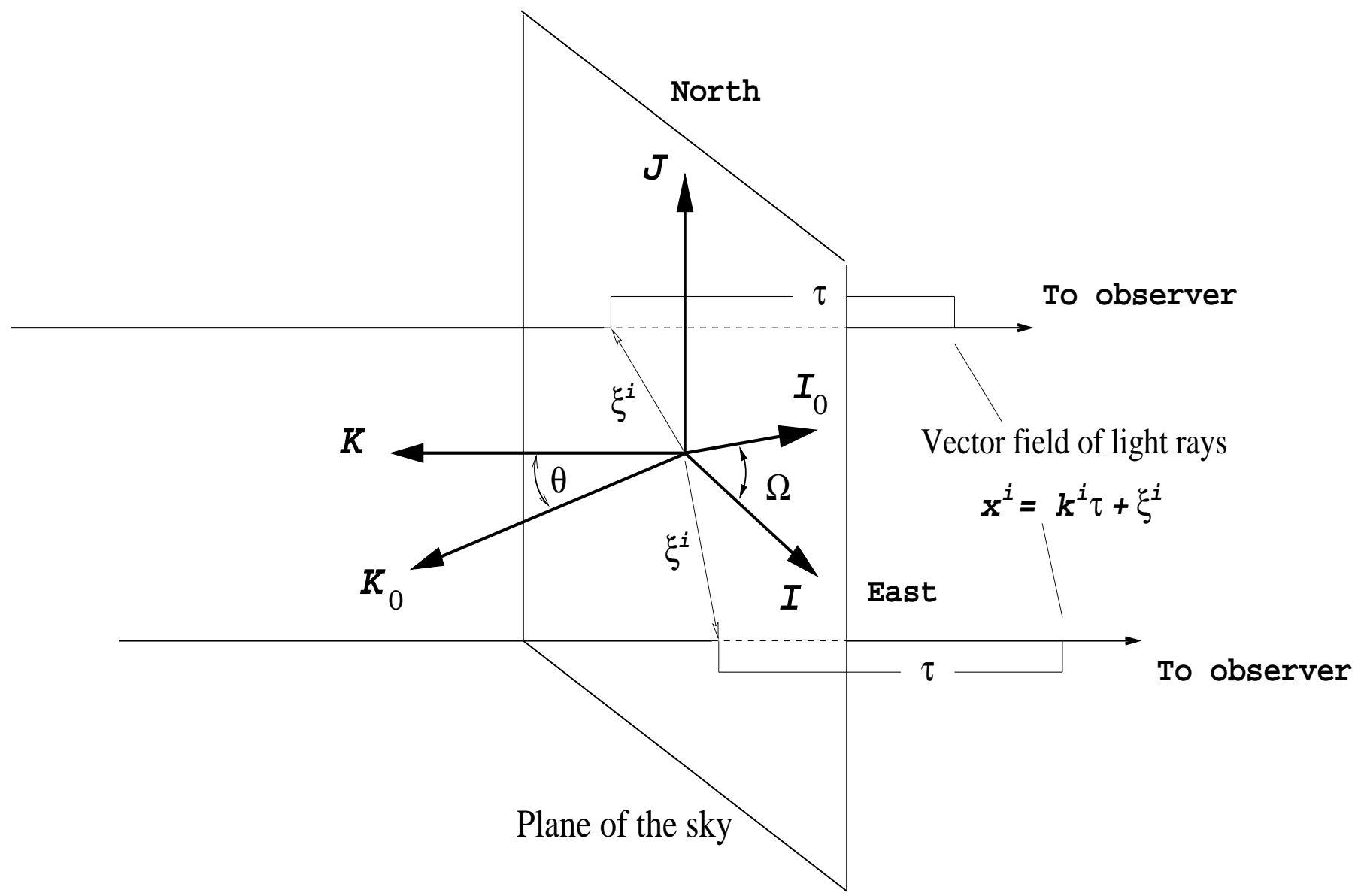

FIG. 1. Astronomical coordinate system used for calculations. The origin of the coordinate system is at the center-of-mass of the source of gravitational waves. The bundle of light rays is defined by the vector field $k^{i}$. The vector $K^{i}=-k^{i}+O\left(c^{-2}\right)$ is directed from observer towards the source of light. The vector $K_{0}^{i}$ is directed from the observer towards the source of gravitational waves. We use in the paper the equalities $K_{0}^{i}=-N^{i}=-x^{i} / r$, where $x^{i}$ are the coordinates of the observer with respect to the source of gravitational waves, and $r=|\mathbf{x}|$. The plane of the sky to the vector $K_{0}^{i}$ is not shown. 


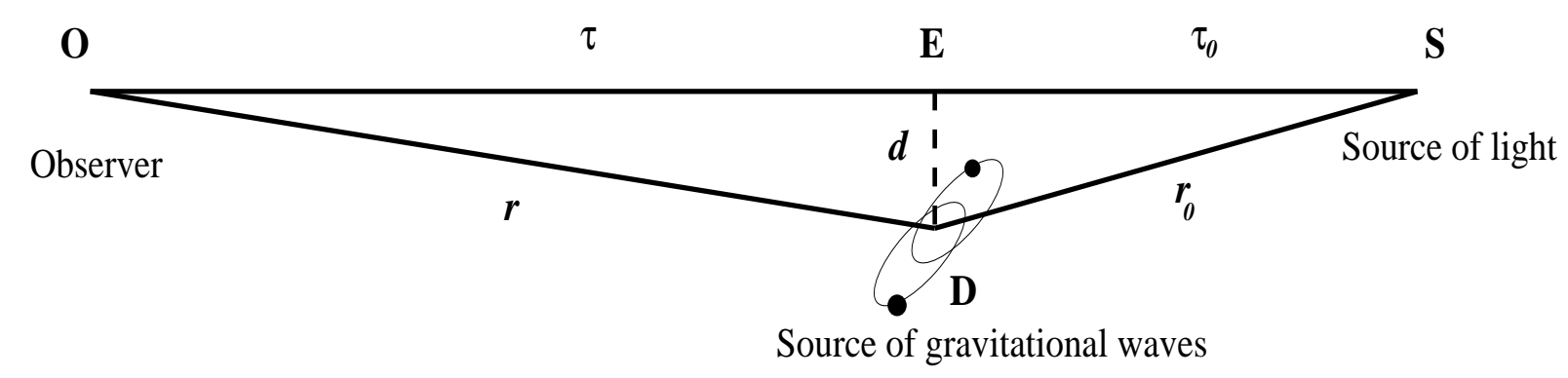

FIG. 2. Relative configuration of observer (O), source of light (S), and a localized source of gravitational waves (D). The source of gravitational waves deflects light rays which are emitted at the moment $t_{0}$ at the point $\mathrm{S}$ and received at the moment $t$ at the point $\mathrm{O}$. The point $\mathrm{E}$ on the line OS corresponds to the moment of the closest approach of light ray to the deflector D. Distances are $O S=R, D O=r, D S=r_{0}$, the impact parameter $D E=d, O E=\tau>0, E S=\tau_{0}=\tau-R<0$. The impact parameter $d$ is small in comparison to all other distances. 


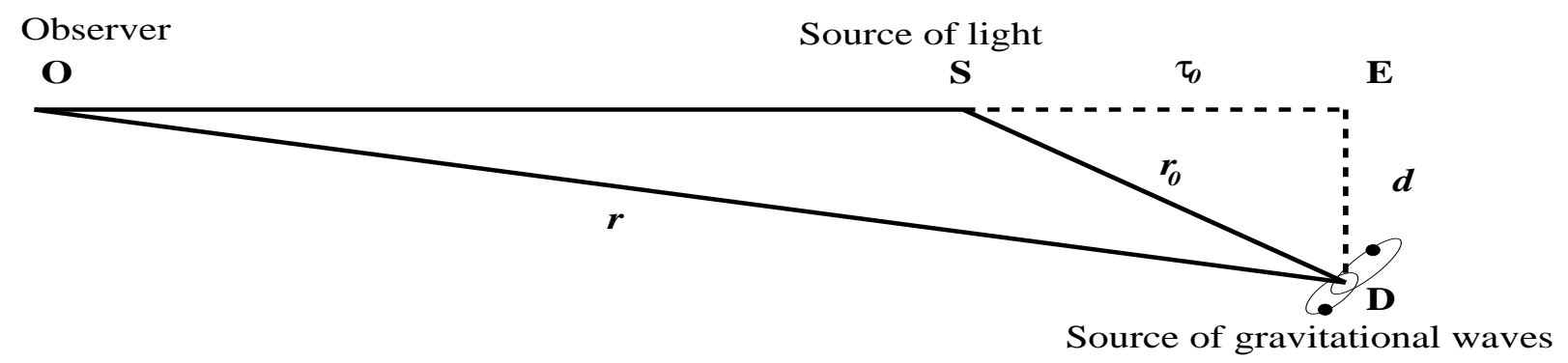

FIG. 3. Relative configuration of observer (O), source of light (S), and a localized source of gravitational waves (D). The source of gravitational waves deflects light rays which are emitted at the moment $t_{0}$ at the point $\mathrm{S}$ and received at the moment $t$ at the point $\mathrm{O}$. The point $\mathrm{E}$ on the line OS indicates the point of minimal distance of the light ray trajectory extrapolated backward to the deflector D. Distances are $O S=R, D O=r, D S=r_{0}$, the impact parameter $D E=d, O E=\tau>0$, $E S=\tau_{0}=\tau-R>0$. The impact parameter $d$ is small in comparision to all other distances. 


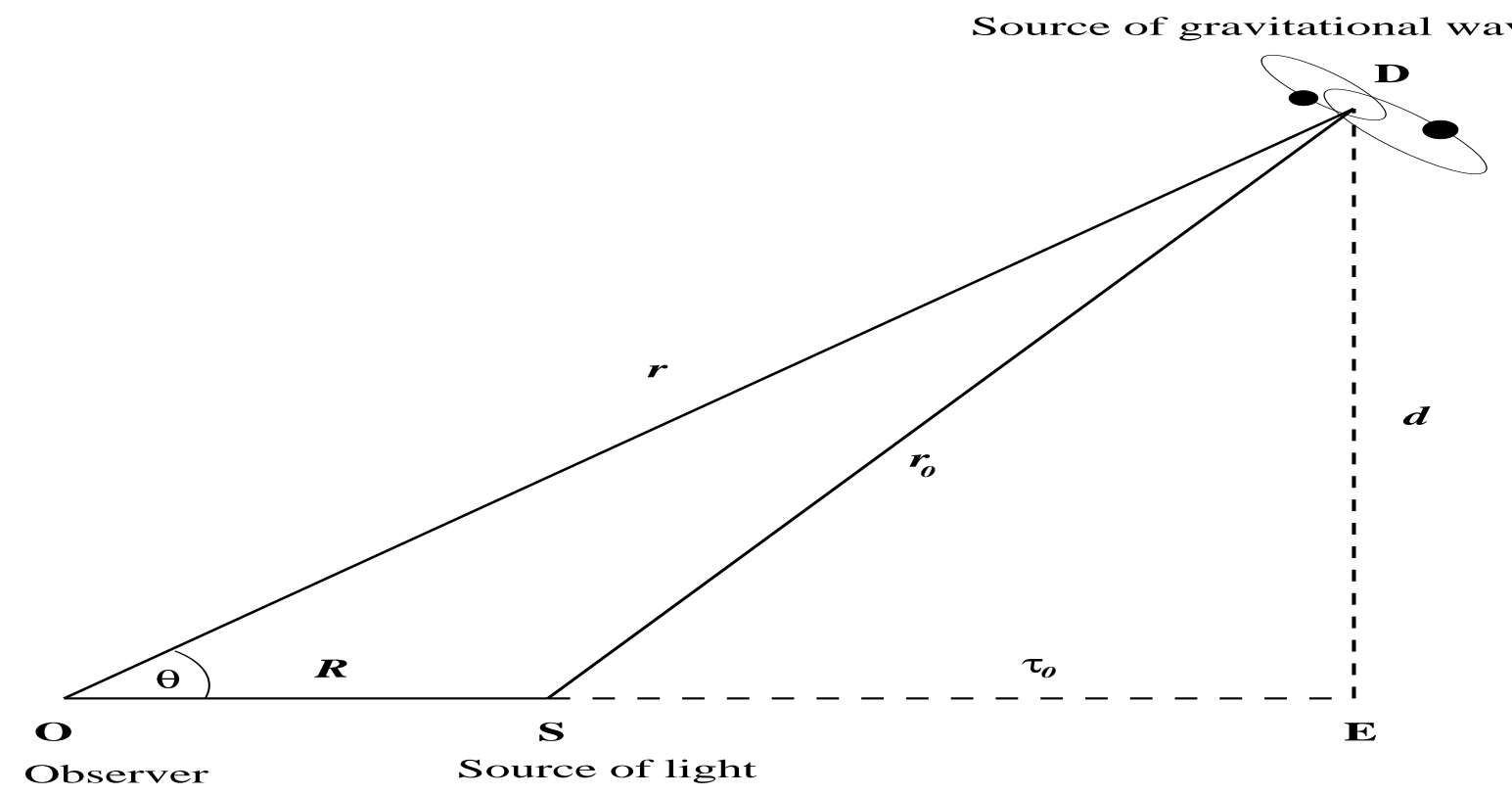

FIG. 4. Relative configuration of observer (O), source of light (S), and a localized source of gravitational waves (D). The source of gravitational waves deflects light rays which are emitted at the moment $t_{0}$ at the point $\mathrm{S}$ and received at the moment $t$ at the point $\mathrm{O}$. The point $\mathrm{E}$ on the line OS corresponds to the moment of the closest approach of light ray to the deflector D. Distances are $O S=R, D O=r, D S=r_{0}$, the impact parameter $D E=d, O E=\tau=r \cos \theta, E S=\tau_{0}=\tau-R$. The distance $R$ is much smaller than both $r$ and $r_{0}$. The impact parameter $d$ is, in general, not small in comparision to all other distances. 